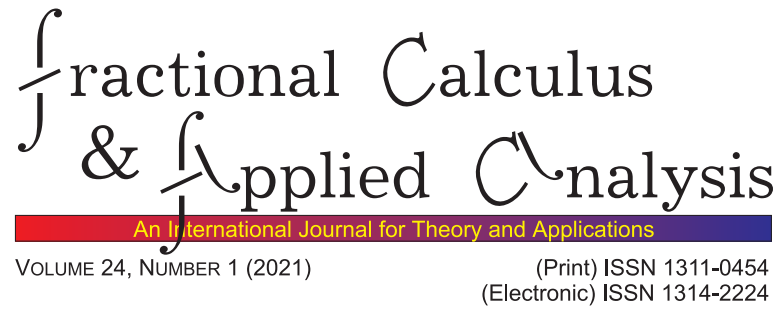

SURVEY PAPER

\title{
RENORMALIZATION GROUP AND FRACTIONAL CALCULUS METHODS IN A COMPLEX WORLD:
} A REVIEW

\author{
Lihong Guo ${ }^{1,2}$, YangQuan Chen ${ }^{2}$, \\ Shaoyun Shi ${ }^{1}$, Bruce J. West ${ }^{3}$
}

\begin{abstract}
The concept of the renormalization group (RG) emerged from the renormalization of quantum field variables, which is typically used to deal with the issue of divergences to infinity in quantum field theory. Meanwhile, in the study of phase transitions and critical phenomena, it was found that the self-similarity of systems near critical points can be described using RG methods. Furthermore, since self-similarity is often a defining feature of a complex system, the RG method is also devoted to characterizing complexity. In addition, the RG approach has also proven to be a useful tool to analyze the asymptotic behavior of solutions in the singular perturbation theory.

In this review paper, we discuss the origin, development, and application of the RG method in a variety of fields from the physical, social and life sciences, in singular perturbation theory, and reveal the need to connect the RG and the fractional calculus (FC). The FC is another basic mathematical approach for describing complexity. RG and FC entail a potentially new world view, which we present as a way of thinking that differs from the classical Newtonian view. In this new framework, we discuss the essential properties of complex systems from different points of view, as well as, presenting recommendations for future research based on this new way of thinking.
\end{abstract}

(c) 2021 Diogenes Co., Sofia

pp. 5-53, DOI: $10.1515 /$ fca-2021-0002

DE GRUYTER 
MSC 2010: Primary 26A33, 81T17; Secondary 82B28, 34A08, 35R11, 60G22, 35B25, 34K26, 34E20

Key Words and Phrases: renormalization group method; singular perturbation; asymptotic solutions; fractional calculus; complexity

\section{Background and motivation}

The concept of a renormalization group (RG) initially derives from quantum field theory, proposed by Stueckelberg and Petermann 110 in 1953, and which proved to be an efficient method of calculation in field theory and statistical physics [14, 45. Meanwhile, in dealing with divergence in perturbed quantum field theory, the renormalization approach is mainly utilized to quench diverging terms before they can destabilize a solution [49. Subsequently, Kadanoff and Wilson et al. [61, 62, 135, 136] applied the RG method to the study of critical phenomena in continuous phase transition processes. Wilson was awarded the Nobel Prize in physics in 1982 for explaining the nature of second-order phase transitions.

Inspired by the applications of the RG method to perturbed quantum field theory and critical phenomena in physics, Chen et al. [23, 24] applied the RG method to singular perturbation theory (SPT), making it an important tool for asymptotic analysis. Although the idea originated in physics, the form of this method completely changed when applied to the treatment of secular terms in SPT.

Subsequently, the domain of application of the RG method was slowly but systematically extended from mechanics to acoustics, into chemistry, branching out into biology and cybernetics, with engineering forays into optimization, and finally into basic mathematical research [40, 58, 92, 106]. In order to better understand the RG method, we discuss its relationships with scaling laws and complex phenomena. This path leads from nonlinear dynamics to chaos theory, to fractal statistics and eventually to the fractional calculus (FC) from different disciplines, each contributing its unique perspective to the understanding of complexity.

1.1. Scaling laws and the renormalization group. Scaling laws, also known as power laws, describe the scale invariance found in many natural phenomena. There are some notable examples that need to be mentioned, since they are drawn from everyday experience. These include Pareto's Law of income distribution [97, whose inverse power law (IPL) form describes the last few percents of a population as controlling most of the income within a Western country; Zipf's IPL of the relative frequency of word usage within a language [84]; and finally the generic example of the IPL form of structural self-similarity of fractals [109, 141]. 
Kadanoff and Wilson established that the self-similarity of dynamic systems near critical points could be described by the RG method. This discovery not only provides a rational and practical theoretical expression for continuous phase transition but also provides a direct physical basis for the RG method. Subsequently, this method proved to be a powerful tool for studying physical phenomena through its treatment of the scaling properties of complex dynamics and chaos [144].

1.2. Complexity and the renormalization group. There is a long history of the inability to describe complex phenomena accurately, with existing contemporary theory leading to empirical paradoxes and confusion [131. The complexity of these phenomena was derived from theory underlying real-world data and they can be seen almost everywhere, such as global climate, fully developed turbulence, financial systems, and the human brain, which makes the study of complexity fascinating.

Although a considerable amount of research has been devoted to the study of complexity, there has not appeared a clear and unified definition of complexity. Therefore, we propose the following two questions to be addressed in this article, and to find at least partial answers:

(1) How can we characterize complexity?

(2) What methods should be used to study complexity in order to understand real-world phenomena better?

There are many definitions of complexity, one being implicit in a phenomenon that is neither entirely ordered nor completely random [126]. Simple ordered systems have patterns that repeat themselves periodically in time or space, and they possess a highly organized structure, that is, a system that can be described by classical Newtonian mechanics. On the other hand, completely disordered systems have a random structure in space or time, described by stochastic dynamics or the random spatial organization of components. Such systems are complicated but simple because they can be easily described using probability density functions (PDFs) so that the main characteristics of the system can be reproduced. The single dynamic trajectory of the former simple system is replaced by an ensemble of trajectories in the later simple system and the behavior by that of the PDF and averages.

However, truly complex systems not only have a large number of small components that interact with non-identical components nearby and their environment, making their behavior inherently challenging to model, but the dynamics are not linear. The resulting chaotic dynamics make the individual trajectories chaotic. Consequently, the dynamics of the ensemble PDF cannot be described by the usual partial differential equations in phase 
space. The equations for the PDF have been determined to be fractional generalizations of the traditional phase space equations [144], as we subsequently discuss. For example, the stock market is a complex system with many interactive properties. Billions of shares and transactions interact through a finite chain of cause and effect, and even if we knew all relevant information, the stock market would still produce unpredictable behavior.

Therefore, the property of complexity in complex phenomena has observable characteristics determined by the interactions, such as nonlinear response to perturbation, long-term memory, scaling laws, and adaptive properties, to name a few. Based on these characteristics, the study of complexity has attracted contributions from many different fields, such as self-organization in physics [55, the spontaneous transition between disorder and order in social sciences [116, 131], the mathematics of nonlinear dynamics (such as chaos) [52, 130], and adaptation in biology [86]. Nevertheless, how to define, much less to measure, a system's complexity remains a controversial topic and is one of the key challenges in studying complex phenomena. Up to now, many quantitative studies of complexity have given rise to a number of measures including, temporal measures, spatial measures, and entropy measures [31, 42, 90.

Inspired by the works of Galanter [42 and West [129], we recreated the trend chart labeled with known measures of complexity. As shown in Fig. 1, when the number of variables in a system is low, certainty, and the dynamics of single-particle trajectories play a leading role, resulting in low levels of complexity. In this few-particle case, behavior can usually be described by well-known techniques, such as celestial dynamics [10, fractals [72], and nonlinear dynamics, including chaos theory [74] and strange attractors [103]. When the number of system variables is high the dynamics of single-particle are replaced by the calculus of PDFs. However, due to the emergence of collective variables, the resulting level of complexity is again low. The treatment of higher levels of complexity arises, moving leftward from the extreme right, using models such as random walks [122, stochastic fractals [77, and the factional probability calculus [132] applied to describe complexity.

The middle region of the mountain range contains a vast region where all the science and mathematics remain to be done. It represents a robust domain where complexity and both the successful and maladaptive networks of society and nature involving both order and randomness, with neither dominating, reside. The delicate balance between order and randomness is observed all around us in living systems, in social organizations, as well as in complex physical networks. For example, the intermittency in the thunderous sound of water from a leaky faucet crashing into the sink 
as you try to get to sleep; the unpredictable tremors that occur in many pathologies of motor control networks; and disruptive interaction between states on the international stage [128]. Note that the trend in the figure implies a nonlinear increase in complexity when moving away from order (extreme left to right) or from disordered (extreme right to left) and is suggested by the rugged terrain of the mountain.

It is the $\mathrm{FC}$ and $\mathrm{RG}$ theory that provide entry into the central region of the unknown. Hopefully, this review will facilitate the passage.

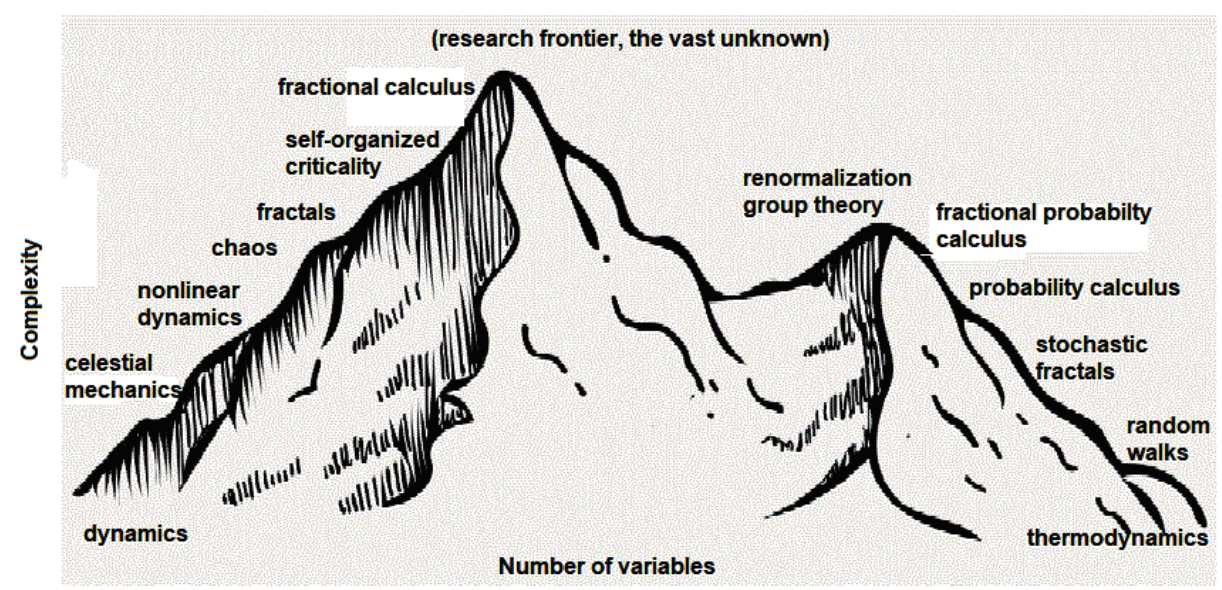

FIgURE 1. The measure of complexity and the number of interacting entities are the axes for rugged landscape of scientific knowledge. The extreme left is described by simple system dynamics, but even here nonlinear dynamics has chaos with more than three degrees of freedom, strange attractors for dissipative nonlinear dynamic systems and nonintegrable Hamiltonians. On the extreme right things are again simple with equilibrium thermodynamics and random walks. Moving from right to left the many degrees of freedom are replaced by low dimensional emergent variables and their mutual interactions which dominate the system.

At the present stage, the research into the nature of complexity divides into two basic strategies [85]. The first strategy is to create and study relatively simple mathematical models, which although they may not accurately capture the behavior of real systems they can abstract the most significant qualitative elements into a solvable framework from which we can extract scientific insights. The research tools used to gain insight into complexity have been nearly as varied as the disciplines to which they 
have been applied, including FC theory [141], RG theory [132], dynamical systems theory [8], game theory [5], information theory [34], and computational complexity theory [85].

In particular, in the process of analyzing complex systems, nonextensive statistical mechanics (NSM) [118, 120, 121 plays an important role in bridging the transitional regions between scale-free and exponential distributions. Nonlinear statistical coupling 82, 83 explains the distinction between scale and the shape of distributions, and that the shape quantifies a nonlinear source. The important distribution families in this paradigm are generalized Pareto distribution and the Student's $t$-distribution.

The second strategy attempts to create more comprehensive, detailed, and realistic models, usually in the form that enables computer simulations, since analytic solutions are often beyond reach. This modelling approach simulates the interacting parts of a complex dynamic network, is accurate in capturing small details, and motivates observation and measurement. For example, Monte Carlo simulation was introduced into the complexity context by [119, especially agent-based simulation [147, 148, in financial markets and other technologies.

1.3. Inverse power laws and fractional calculus. One of the first analytic treatments of the generation and spreading of complexity was the movement of heavy particles in an ambient fluid of lighter particles. Today we would classify this behavior as complicated but not complex. Consider the standard diffusion equation:

$$
\frac{\partial \rho(x, t)}{\partial t}=D \frac{\partial^{2} \rho(x, t)}{\partial x^{2}}
$$

where $D$ denotes a positive constant, $x$ and $t$ are the space and time variables, respectively, and $\rho(x, t)$ is the phase space density function. The diffusion equation is used to describe the change of the matter density in diffusive phenomena. In physics, Eq. (1.1) describes the macroscopic behavior of many large particles undergoing Brownian motion in an ambient fluid and is often replace by the PDF $P(x, t)$. In mathematics, the PDF is related to Markov processes, and is often modeled using simple random walks. The diffusion model can also be applied in many other fields, such as the movement of alleles in population genetics [80, or more generically the flow of information in complicated networks [129].

One of the basic boundary-value problems for diffusion is the Cauchy problem:

$$
P\left(x, 0^{+}\right)=\delta(x), \quad-\infty<x<+\infty ; \quad P(\mp \infty, t)=0, \quad t>0,
$$

where $\delta(x)$ is the Dirac delta function. This initial value problem describes a point source for a tracer released at the origin at time $t=0$, which then 
diffuses throughout the ambient fluid. The formal solution to the Cauchy problem is:

$$
P(x, t)=\int_{-\infty}^{\infty} \mathcal{G}\left(x-x^{\prime}, t\right) P\left(x^{\prime}, 0^{+}\right) \mathrm{d} x^{\prime},
$$

where the Green's function $\mathcal{G}(x, t)$, also known as the propagator, is obtained by taking the Fourier transform of Eq. (1.1), solving the resulting linear rate equation for the characteristic function and inverse Fourier transforming the resulting solution to obtain:

$$
\mathcal{G}(x, t)=\mathcal{F} \mathcal{T}^{-1}\left\{e^{-|k|^{2} \sigma^{2}} ; x\right\}=\frac{1}{\sqrt{2 \pi} \sigma} e^{-x^{2} /\left(2 \sigma^{2}\right)}, \quad \sigma^{2}=2 D t,
$$

which when inserted into Eq. (1.2) yields the well-known Gaussian or normal PDF.

An example of the FC is introduced in this context by replacing the second-order space derivative with a symmetric space-fractional derivative of order $\beta$ with $0<\beta \leqslant 2$. We can write the symmetric space-fractional diffusion equation [50]:

$$
\frac{\partial P(x, t ; \alpha)}{\partial t}=D_{0} \frac{\partial^{\beta} P(x, t ; \alpha)}{\partial|x|^{\beta}}, \quad x \in \mathbb{R}, \quad t \in \mathbb{R}^{+},
$$

where $D_{0}$ is a positive coefficient and the fractional derivative is taken in the sense of Riesz (for details, see Appendix A). Here again the Fourier transform of the PDF yields the equation for the characteristic function with the solution from the Fourier transform of Eq. (1.3) given by the inverse Fourier transform of the characteristic function:

$$
P(x, t ; \beta)=\int_{-\infty}^{\infty} e^{i k x} e^{-D_{0} t|k|^{\beta}} \frac{\mathrm{d} k}{2 \pi} .
$$

This is a symmetric Lévy PDF, the solution to the Cauchy initial value problem for the fractional diffusion equation given for Eq. (1.3). Note that the only closed-form solutions given by Eq. (1.4) arise for a limited number of values of the Lévy index in the interval $0<\beta \leqslant 2$, including that of Gauss $\beta=2$. The Gauss PDF is the only case having finite second moments, all other second moments of the Lévy PDF diverge including the Cauchy with $\beta=1$. The diverging second moment violates a fundamental condition necessary for the proof of the central limit theorem (CLT) to converge. Such strange behavior is common in our complex world.

It is worth mentioning here that as $|x| \rightarrow \infty$, the analytic solution of the symmetric Lévy PDF can be obtained by taking the $|k| \rightarrow 0$ limit of 
the characteristic function within the integrand of Eq. (1.4):

$$
\lim _{|x| \rightarrow \infty} P(x, t ; \beta) \propto \frac{1}{x^{\beta+1}} .
$$

Thus, the asymptotic form of the symmetric Lévy PDF is an IPL [25, 127. Using the IPL the failure of the second moment to converge for $0<\beta<2$ is obvious.

1.4. Renormalization group and fractional calculus. From the perspective of scaling laws or IPLs, the present review seeks connections between the RG and the FC. Furthermore, we find that the two research areas are not independent of one another, but have a particular relationship, in that they both emerged from studies seeking to understand complex phenomena. As mentioned above, these two mathematical strategies provide systematic ways to research complexity, and have the potential of providing deep understanding of actual phenomena. Based on this, the purposes of this review are as follows:

(1) Introduce the RG and FC methods and point out how they are connected.

(2) Provide new ideas for the future improvement and application of these methods.

Section 2 briefly introduces the RG method, which although derived from mathematical physics has applications in a broad spectrum of disciplines. We touch on a number of these non-physical application in this section. Section 3, we discuss the origin and developments of the RG method in SPT and its applications in various fields of disciplinary research. In Section 4, the connection between RG and FC is discussed. In the last section, conclusions and prospects are given.

\section{The origin and development of renormalization group methods}

As mentioned earlier, the concept of RG was first proposed by Stueckelberg and Petermann [110] well over half a century ago. Since its inception it was known that the $\mathrm{RG}$ is not a group in the strict mathematical sense. It is, in general, a semigroup, because its inverse elements cannot be defined [48]. As a mathematical method, the $R G$ theory was initially designed to solve problems having self-similar structures with an infinite range, such as in phase transitions [48] and critical phenomena [3]. However, in some complex physical processes, it is difficult to grasp the details of the internal structure and interaction as part of the research objective. Therefore, from a symmetry perspective, the RG method is an important research 
method to study the self-similar structures of physical phenomena and the invariable properties under symmetric transformations.

2.1. Basic ideas. The physical phenomena studied by the RG method have one common feature: the correlation length diverges $\xi \rightarrow \infty$, that is, there is no characteristic scale with which to characterize the process. This is observed during a phase transition of water to ice as the temperature $T$ is lowered to its critical value $T_{c}$. The water molecules have a shortrange exponential correlation function and a constant correlation length when $T>T_{c}$, but as $T$ approaches $T_{c}$ the correlation function becomes a long-range IPL and the correlation length diverges as the water solidifies.

The purpose of the RG method is to derive typical scaling relations among the physical parameters controlling the phenomenon's behavior:

$$
f\left(K_{1}, K_{2}\right)=K_{1}^{-\alpha} F\left(\frac{K_{2}}{K_{1}^{\tau}}\right),
$$

where $K_{1}, K_{2}$ are coupling constants within the system and $F(\cdot)$ is an unknown analytic function. For example, for ferromagnetic systems, $K_{1}^{-1}$ could be the system scale size and $K_{2}$ is the system temperature; for hydrodynamic systems, $K_{1}$ could be the wave numbers and $K_{2}$ the frequency. The scaling indices $\alpha$ and $\tau$ are the critical exponents of the system. When the correlation length diverges $\xi \rightarrow \infty$, it is found that the above scaling relation only depends on the symmetry of the system but is independent of the micro details, and possesses universality. This emergence of universal macroscopic behavior was the theme of the classic Science article "More is different" by P. Anderson [4] who received the Nobel Prize in Physics.

As mentioned in Section 1.1, the scaling laws refers to the fact that systems at different scales have the same properties. At the same time, due to the correlation length $\xi$ diverging to infinity, the universality signifying the individuality of different systems retreats to secondary importance, and its common behavior of universality emerges center stage.

In addition to the theoretical analysis alluded to above, experimental observations [53, 104] found that different physical systems do have similar characteristics near the critical points. The phenomenon that occurs near the continuous phase transition points (critical points) is called a critical phenomenon. The characteristics near these points are summarized in scaling laws and in the universality hypothesis (see Fig. 2).

The basic idea of the universality hypothesis is that the long-range correlations near the critical points determine all the singularity properties of the underlying process. When the state of the system is close to its critical points, where the correlation length diverges $(\xi \rightarrow \infty)$, we can obtain the same results by describing physical phenomena on any finite 


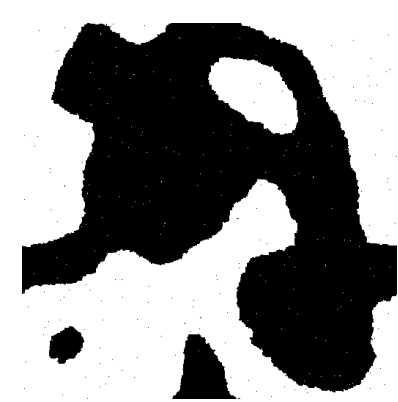

(a) $\mathrm{T}=0.5 \mathrm{~T}_{c}$

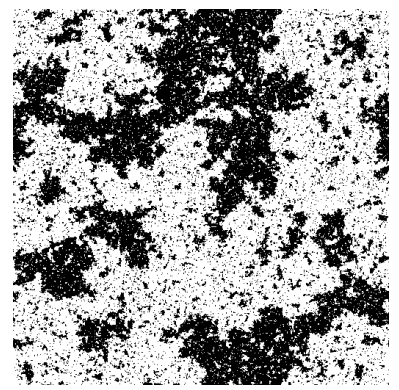

(b) $\mathrm{T}=\mathrm{T}_{c}$

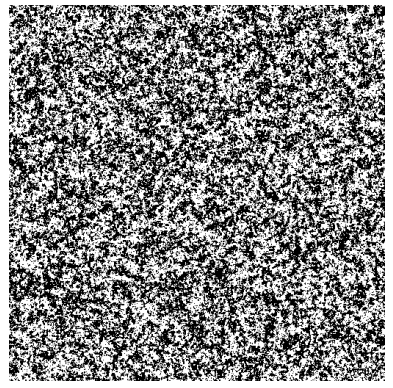

(c) $\mathrm{T}=1.28 \mathrm{~T}_{c}$

FiguRE 2. Ising model simulations of a dynamic system at critical and non-critical temperatures. (a) Binary $256 \times$ 256 lattices showing the configuration of spins after 2,000 timesteps at low temperature $\mathrm{T}=0.5 \mathrm{~T}_{c} ;(\mathrm{b})$ at critical temperature $\mathrm{T}=\mathrm{T}_{c} ;(\mathrm{c})$ at high temperature $\mathrm{T}=1.28 \mathrm{~T}_{c}$. At high temperature the spins are randomly configured, at low temperature they have an alignment of spins, and at critical temperature they have a fractal configuration.

Link: https://github.com/mattbierbaum/ising.js

scale near the critical points. Similar to isometric spirals of different scales by zooming in and out, the textures can be superimposed together.

Due to the above scaling characteristics, the RG method can be applied to the analysis of critical phenomena. The small-scale fluctuations are smoothed by the scale transformation, and the effective action at the largescales dominate. Since the similarity between global and local behavior has been achieved, this transformation will not affect the global properties.

The main steps of the RG method are as follows:

(1) Use the renormalization transformation to obtain a coarsegrained operator by changing the length scale and reducing the number of degrees of freedom acting on the state variable.

(2) Induce the transformation of the model parameters. Generally, use the approximate method to keep the partition function of the system consistent. In this process, we can obtain the renormalization flow (RG flow).

(3) Analyze the fixed points of this transformation, as well as the behavior of the system near the fixed points. 
2.2. Renormalization group transformations. Much of the analytic work in physics is done using conservative Hamiltonian systems, in large part because such systems reproduce the mechanical forces of Newton. Let $H=H(\mathbf{K})$ be the Hamiltonian of a system of interest, where $\mathbf{K}=$ $\left(K_{1}, K_{2}, \cdots\right)$ is the vector of coupling constants. Each set of these constants defines a point in the "Hamiltonian space", that being the space of coupling constants. The RG transformations operate on this space:

$$
\mathbf{K}^{\prime}=\mathcal{R}(\mathbf{K}) .
$$

The RG operator $\mathcal{R}$ reduces the number of degrees of freedom from $N$ to $N^{\prime}$ :

$$
b^{d}=\frac{N}{N^{\prime}},
$$

where $b$ is the rescaling factor, and $d$ is the spatial dimension.

The essential condition to be satisfied by the RG transformation is that the form of the partition function remains invariant under the transformation:

$$
Z_{N^{\prime}}\left(\mathbf{K}^{\prime}\right)=Z_{N}(\mathbf{K})
$$

Therefore the total free energy does not change, but the free energy per unit cell (spin) increases as:

$$
f\left(\mathbf{K}^{\prime}\right)=b^{d} f(\mathbf{K}) .
$$

All lengths, measured in units of the new lattice spacing, are reduced by the factor $b$. Thus, the correlation length scale is:

$$
\xi^{\prime}=\frac{1}{b} \xi .
$$

Fixed points. The iterations of the $\mathrm{RG}$ transform $\mathcal{R}$ traces a trajectory in the Hamiltonian space, this "Hamiltonian flow" is identified as RG flow. The most important information regarding RG flow concerns its fixed points. The trajectories end at the fixed points $\mathbf{K}^{*}$, defined by the $\mathrm{RG}$ transform becoming equivalent to the identity operator:

$$
\mathbf{K}^{*}=\mathcal{R}\left(\mathbf{K}^{*}\right),
$$

which occurs, if and only if:

$$
\mathbf{K}^{\prime}=\mathbf{K} \equiv \mathbf{K}^{*} .
$$

The set of fixed points provides the possible macroscopic state of the system at a large scale. At these points the system is invariant under subsequent scale changes and thus the correlation length remains constant:

$$
\xi^{\prime}=\xi \equiv \xi^{*},
$$

and does not change. 
We can regard the $\mathrm{RG}$ flow as evolution such as that of a dynamical system. In particular, there are some fixed points in space, which represent the particular theory of scale invariance. These fixed points can be divided into two categories: stable and unstable critical points. When a fixed point has no unstable directions they are stable critical points and are called "sinks". If we start from a point in space and approach such an attractive fixed point, the RG flow will be brought closer to the fixed point by the behavior described using effective long-wave theory. When a fixed point has unstable directions in space they are unstable critical points and are called "sources".

Therefore, the asymptotic behavior of all correlation functions in the original system are determined by the nature of the fixed points. These points divide the space into different regions and describe the transition between different phases. This is the bare mathematics of critical phenomena.

An example: To illustrate the main ideas of the RG method, we use a classical model from statistical physics - the two-dimensional Ising model. The model consists of a square lattice with $N$ sites and $E$ nearest neighbor bonds, the $i$-th site of the lattice is assigned a spin $s_{i}, 1 \leqslant i \leqslant N$, which can be +1 (spin up) or -1 (spin down). When neighboring spins are oriented in the same (different) direction, there is an interaction energy $-J(J)$, where $J$ is the spin-spin coupling constant. The spin $s_{i}$ also couples with an external magnetic field $h$ with an energy $-h s_{i}$. In this case, both the height and width of the blocks of spins should have the same length $b a$, where $b$ is the rescaling factor, and $a$ is the lattice spacing (see Fig. 3).

Thus the Hamiltonian (total energy) of the spin system can be written as:

$$
H(\mathbf{s})=-J \sum_{(i, j)} s_{i} s_{j}-h \sum_{i}^{N} s_{i}
$$

where $\mathbf{s}$ represents the configuration of $N$ spins, i.e., $\mathbf{s}=\left(s_{1}, \cdots, s_{N}\right)$, the first summation is over all $E$ nearest-neighbor pairs of spins and the second summation is over all $N$ sites of the lattice. According to statistical mechanics, at a given absolute temperature $T$, the probability for $\mathbf{s}$ to appear $p(\mathbf{s})$ is proportional to the Boltzmann factor $\exp (-\beta H(\mathbf{s}))$, and thus is given by:

$$
p(\mathbf{s})=\frac{\exp (-\beta H)}{Z_{N}}=\frac{\exp \left(K \sum_{(i, j)} s_{i} s_{j}+B \sum_{i}^{N} s_{i}\right)}{Z_{N}},
$$

where $\beta=1 /\left(k_{B} T\right), k_{B}$ is the Boltzmann constant, $K=\beta J, B=\beta h$, and:

$$
Z_{N}=\sum_{\left(s_{1}, \cdots, s_{N}\right)} \exp (-\beta H)
$$

is the partition function of the system. 

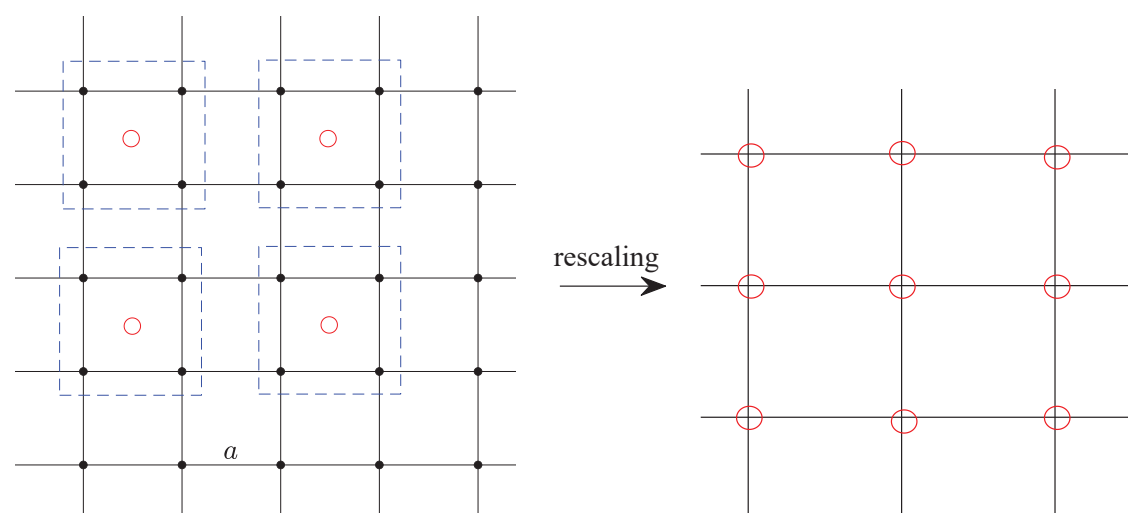

FiguRE 3. Block spins transformation. The blocks (dotted blue box) behave as if they were atoms, but with a reduced number. • represents the lattice spin, o represents the block spin.

One way of defining the spin of a block is to use the majority rule:

$$
s_{I}=\operatorname{sgn}\left(\sum_{i \in I} s_{i}\right)
$$

where $I$ refers to the index of the blocks. Consequently, the Hamiltonian for the coarse-gained problem is given approximately by:

$$
H^{\prime}\left(\mathbf{s}_{\mathbf{I}}\right)=-J^{\prime} \sum_{(I, J)} s_{I} s_{J}-h^{\prime} \sum_{I}^{N^{\prime}} s_{I} .
$$

Then the coarse-gained operator for the spin configuration induces a transformation in the space of model parameters:

$$
\mathbf{K}^{\prime}=\mathcal{R}(\mathbf{K})
$$

where $\mathbf{K}=(K, B)$. This example constitutes the block renormalization transformation.

Lattice spin models are not restricted to the understanding of phase transitions and critical phenomena in physical systems. Versions of this model have been applied to fractal geometry, such as the criticality and phase transitions in Koch curves [113] and Sierpinski gaskets [44, as well as to the molecular theory of biological evolution and the origin of life [29, 37] and finally in the use of network science to the decision making behavior of humans [128].

The above briefly describes the main ideas in the derivation of RG theory, so let us now turn our attention toward applications. The applications 
of the RG method in various disciplines both past and present has led to extensions and nuanced interpretations of the RG.

\subsection{Applications of renormalization group.}

2.3.1. Quantum electrodynamics. Gell-Mann and Low 45] introduced the RG into quantum electrodynamics in 1954. In 1970, Callan [17] and Symanzik 114 proposed the well-known Callan-Symanzik equation which describe the evolution of the $n$-point correlation functions under variation of the energy scale $n$ in this context.

2.3.2. Condensed matter physics. Kadanoff [61] and Wilson [133, 134] introduced the RG into the study of condensed matter physics in the decade of the seventies. Kadanoff is often referred to as the person who did not receive the Nobel prize in physics for his intuitively appealing scaling of the Hamiltonian presented herein, which is found in virtually every text on the subject of critical phenomena.

Wilson interpreted the self-similar nature of critical phenomena to imply that the renormalization equations from quantum field theory can be used in the study of critical phenomena. All physical properties near the critical points are independent of the local details and the same behavior can be obtained by selecting a description on any scale. Hence the theory of critical phase transitions was established, and the RG method has subsequently been successfully applied to statistical physics and related fields.

The frontiers of knowledge have been pushed back in a number of areas using this method, such as in the understanding of critical phenomena 3] and phase transitions [48, as well as, in the development of a grand unified theory [89].

2.3.3. Fermion interaction system. Solyom [107] applied the RG method to study the infrared divergence of one-dimensional Fermi gas in 1979. Subsequently, the RG method has become a significant research method for dealing with fermion interaction systems. This method became widely used in the study of classical phase transitions and the critical behavior of quantum phase transitions in one-dimensional and higher-dimensional Fermi systems [39, 41, 98, in providing insight into quantum impurity problems [63, 125], and non-equilibrium state of Fermi system [101, 102, as well as many other physical problems.

2.3.4. Complex networks. The RG method has proven invaluable, not just in physics, but has applications in the field of complex networks [12, 112. As stated by Barabási and Bonabeau [9], many real world complex networks, including the World Wide Web (WWW), social networks, protein interaction networks, and cellular networks, are self-similar under a certain 
length-scale transformation, that is, the number of links per node follows an IPL PDF.

Song et al. [43, 108, generalized the box-counting method to complex networks, and pointed out that for many complex networks, there is a selfsimilarity index related to the fractal dimension, which possesses inherent self-similarity properties. Furthermore, by adopting the RG ideas reveals that the similarity and scale-free degree IPL PDFs are established in all coarse-grained stages of the network. The degree distribution $P\left(k^{\prime}\right)$ of the renormalization network is invariant under the RG transform:

$$
\mathcal{R} P(k)=P\left(k^{\prime}\right) \approx\left(k^{\prime}\right)^{-\gamma},
$$

where $P(k)$ is the $\mathrm{PDF}$ of the $k$ links per node, and the degree exponent lies in the interval $2<\gamma<3$.

Hernández-Bermejo [56] used the RG method to study a class of complex metabolic network models. Their study showed that the accuracy of the reported IPL models over several orders of magnitude of the metabolite concentration because the IPLs are the invariant solutions to the RG equations.

2.3.5. Time series. Time series are a set of data points, typically consisting of events arranged in order of their time of occurrence. Usually, the intervals of a time series are of constant value (such as one second, five minutes), so the time series can be analyzed and processed as discrete-time data. Examples of time series are gross domestic product (GDP), consumer price index (CPI), weighted stock price index, interest rate, and exchange rate. Or selecting from an equally complex set of data, we can mention the intermittency of heart rate intervals, stride lengths in walking, or EEG data.

Time series are widely used in mathematical statistics, signal processing, pattern recognition, econometrics, and most applications involving time data measurements in science and engineering. Therefore, the analysis and prediction of time series is the focus of much research in these fields.

A time series prediction and analysis algorithm based on the RG transformation proposed in [6, 142] was different from traditional prediction models, such as ARIMA and GARCH [115]. RG self-similarity does not seek to discover the relationship between historical points, but rather attempts to identify the dynamic trends that lead to these points. This algorithm was applied to predict financial time series one step ahead, and to the future development direction of financial time series.

2.3.6. Probability theory. In classical probability theory, the CLT occupies a significant position. This theorem establishes that under well-defined 
conditions, the mean values of a large number of independent, identicallydistributed (i.i.d) random variables, with finite mean and variance, converge to a normal distribution after appropriate standardization. The CLT is the theoretical basis of traditional mathematical statistics as well as error analysis and points out that the sum of a large number of random variables approximately obeys a normal distribution.

There are different methods for proving the CLT. The derivation of the CLT by the RG method can necessarily reveal its universal result, that is, the normal distribution is the fixed point of the sum of these random variables under the action of RG operator [30, 60. Besides, the generalized CLT can also be proved by using this point of view, such as a large number of i.i.d random variables with infinite variance, converging to Lévy stable distribution under appropriate scaling [2, 18, 19].

Consider the dynamic variable $Z(t)$ having the homogeneous scaling behavior:

$$
Z(\lambda t)=\lambda^{\delta} Z(t)
$$

where $\lambda$ is a parameter and $\delta$ is a scaling index. In the phase space for the system the dynamic variable is replaced with the trajectory mapped out by $(z, t)$ and $P(z, t) \mathrm{d} z$ is the probability that the random variable lies in the interval $(z, z+\mathrm{d} z)$ at time $t$. The scaling behavior expressed in Eq. (2.1) is manifest in the scaling of the PDF:

$$
P(z, t)=\frac{1}{t^{\delta}} F\left(\frac{z}{t^{\delta}}\right)
$$

where $F(\cdot)$ is an unknown analytic function of the scaled variable. When the scaling index is $\delta=1 / 2$ this corresponds to the Gauss distribution. When the PDF is that of Lévy the scaling index is given by $\delta=1 / \beta$ and $\beta$ is the Lévy index from Eq. (1.4). We subsequently show that Eq. (2.2) is the fixed point RG solution of the general fractional equation:

$$
\partial_{t}^{\alpha}[P(z, t ; \alpha, \beta)]=K \partial_{|z|}^{\beta}[P(z, t ; \alpha, \beta)],
$$

and $\delta=\alpha / \beta$ [129]. There are two kinds of non-locality in the fractional equation for the PDF in Eq. (2.3): the Caputo derivative in time on the left introduces memory into the evolution; the Reisz derivative in space on the right introduces non-locality in space into the evolution. The RG solution to this fractional evolution of the PDF given by Eq. (2.2) ties the scaling in space and time together through the ratio of the two fractional indices.

Let us now turn our attention to presenting some details on the origin, developments, and applications of the RG method in SPT. 


\section{Renormalization group methods in singular perturbation theory}

Perturbation theory includes mathematical methods that were initiated to obtain asymptotic analytic solutions to mathematical physics problems. In general, these problems were regarded as systems with small perturbations to the parameters or structures of ideal dynamic models. This perspective evolved and today there are two different types of perturbation problems: regular and singular. When the asymptotic expansion uniformly approaches an exact solution, the perturbation problem is regular. The solutions to the equations of this system's dynamics, with the perturbation parameters set equal to zero can approximately replace the solutions of the original system. This is called the degenerate system. Otherwise, it is a singular perturbation problem. In the latter case the solution to the degenerate system's equations of motion is substantially different from the solution to the equation for the original system.

The study of the singular perturbation problem has developed into an essential branch of control theory. The commonly used techniques include the method of multiple scales, boundary layers, asymptotic matching, Wentzel-Kramers-Brillouin (WKB) approximation, stretched coordinates, and the averaging method, as mentioned in 24, 81. However, it should be noted that all the methods mentioned above have their shortcomings, which hinder their application to real-word mechanical systems [81. Therefore, the authors of [20, 21, 22, 23, 24 proposed a new method based on RG ideas to overcome these shortcomings.

3.1. The origin. From the perspective of the applications of the RG method in perturbed quantum field theory and critical phenomena, Chen et al. in the years from 1991 to 1996 [20, 21, 22, 23, 24 demonstrated a unified understanding of various singular perturbation methods and reductive perturbation methods using several examples. Firstly, they discussed the general relation between the RG method and multiple-scale analysis by means of examples. Secondly, for a class of singular perturbation problems, they showed that these problems could also be solved by the RG method. Thirdly, they demonstrated with several switchback problems that the RG approach has technical advantages over conventional asymptotic methods. Lastly, they demonstrated that the RG method is a general and systematic method to derive slow-motion equations using the Newell-Whitehead equation.

To better understand their work, and in keeping with historical precedent we use an example to illustrate the efficiency of this method. 
Rayleigh equation. Lord Rayleigh supplemented the weak viscous force acting on a harmonic oscillator with a cubic nonlinearity, which in standard notation can be expressed as:

$$
\begin{aligned}
& \frac{\mathrm{d}^{2} y(t)}{\mathrm{d} t^{2}}+y(t)=\varepsilon\left[\frac{\mathrm{d} y(t)}{\mathrm{d} t}-\frac{1}{3}\left(\frac{\mathrm{d} y(t)}{\mathrm{d} t}\right)^{3}\right], \\
& y\left(t_{0}\right)=0, \quad \frac{\mathrm{d} y\left(t_{0}\right)}{\mathrm{d} t}=R_{0},
\end{aligned}
$$

where $0<\varepsilon \ll 1$ is known as the small perturbation parameter. The solution to this equation is obtained by assuming a naive expansion in powers of $\varepsilon$ :

$$
y(t)=y_{0}(t)+\varepsilon y_{1}(t)+\varepsilon^{2} y_{2}(t)+\cdots,
$$

which when substituted into the Rayleigh equation, and comparing coefficients of the same power of $\varepsilon$ yields:

$$
\begin{aligned}
& \varepsilon^{0}: \frac{\mathrm{d}^{2} y_{0}(t)}{\mathrm{d} t^{2}}+y_{0}(t)=0, \\
& \varepsilon^{1}: \frac{\mathrm{d}^{2} y_{1}(t)}{\mathrm{d} t^{2}}+y_{1}(t)=\frac{\mathrm{d} y_{0}(t)}{\mathrm{d} t}-\frac{1}{3}\left(\frac{\mathrm{d} y_{0}(t)}{\mathrm{d} t}\right)^{3}, \\
& \ldots
\end{aligned}
$$

This subsidiary set of equations is solved in ascending orders of the perturbation parameter. The solution to the oscillator equation is inserted as the driver to the order $\varepsilon$ equation and solved. In this way we obtain the straightforward perturbation solution to $O(\varepsilon)$ of the Rayleigh equation:

$$
\begin{aligned}
y(t)= & R_{0} \sin \left(t+\theta_{0}\right)+\varepsilon\left[-\frac{R_{0}^{3}}{96} \cos \left(t+\theta_{0}\right)+\frac{R_{0}}{2}\left(1-\frac{R_{0}^{2}}{4}\right)\left(t-t_{0}\right)\right. \\
& \left.\sin \left(t+\theta_{0}\right)+\frac{R_{0}^{3}}{96} \cos 3\left(t+\theta_{0}\right)\right]+O\left(\varepsilon^{2}\right),
\end{aligned}
$$

where $\theta_{0}$ is a constant determined by the initial conditions at $t=t_{0}$.

Since this direct expansion possesses $\frac{R_{0}}{2}\left(1-\frac{R_{0}^{2}}{4}\right)\left(t-t_{0}\right)$ which is the so called secular term [81]. The secular terms make $y_{n}(t) / y_{n-1}(t)$ unbounded as $t$ approaches to infinity, such as $t^{n} \cos t$ and $t^{n} \sin t$. Therefore, the solution (3.1) is divergent and not uniformly valid. To regularize the series, which is a formal way of suppressing the divergence in the solution, we introduce an arbitrary intermediate time $\tau$ that partitions $t-t_{0}$ into $t-\tau$ and $\tau-t_{0}$. We absorb the terms containing $\tau-t_{0}$ into the renormalized 
counterparts $R$ and $\theta$ as follows:

$$
\begin{aligned}
R & =R_{0}+\varepsilon \frac{R_{0}}{2}\left(1-\frac{R_{0}^{2}}{4}\right)\left(\tau-t_{0}\right), \\
\theta & =\theta_{0} .
\end{aligned}
$$

This partitioning enables us to rewrite Eq.(3.1) after some algebra as the following renormalized perturbation result:

$$
\begin{aligned}
y(t)= & {\left[R+\varepsilon \frac{R}{2}\left(1-\frac{R^{2}}{4}\right)(t-\tau)\right] \sin (t+\theta)-\varepsilon \frac{R^{3}}{96}[\cos (t+\theta)} \\
& -\cos 3(t+\theta)]+O\left(\varepsilon^{2}\right),
\end{aligned}
$$

where $R$ and $\theta$ are functions of $\tau$. Since $\tau$ does not appear in the original problem, the solution should not depend on it. Therefore, requiring that the solution be independent of $\tau$ yields:

$$
\left.\frac{\partial y}{\partial \tau}\right|_{\tau=t}=0
$$

for any $t$, so that the RG equation is:

$$
\begin{aligned}
& \frac{\mathrm{d} R}{\mathrm{~d} t}=\varepsilon \frac{1}{2} R\left(1-\frac{1}{4} R^{2}\right)+O\left(\varepsilon^{2}\right), \\
& \frac{\mathrm{d} \theta}{\mathrm{d} t}=O\left(\varepsilon^{2}\right) .
\end{aligned}
$$

Thus, the final uniformly valid result is

$$
y(t)=R(t) \sin t+\frac{\varepsilon}{96} R(t)^{3}[\cos (3 t)-\cos t]+O\left(\varepsilon^{2}\right),
$$

where the time-dependent amplitude $R(t)$ is given by the solution to the nonlinear rate equation Eq. (3.2).

The idea behind the RG method applied in this way is that the resonant part of the equation dominates the dynamics of an system. By decomposing the nonlinear term into resonant and non-resonant parts, the former is constant in time and yields a secular term, which leads to the RG equation. The solution to the RG equation is the main piece of an approximate solution to the initial perturbed equation. Such a decomposition is very useful in studying the long-time behavior of solutions. The RG method became a powerful technique with which to solve a sequence of perturbation problems. The advantage of this method over other more algebraically intensive methods is that the starting point is a straightforward naive perturbation expansion, for which no prior knowledge is required. In contrast to conventional methods, this approach neither requires assumptions regarding the perturbation series' structure nor does it require matching of the solution pieces in an asymptotic domain. 
To illustrate the effects of this method theoretically, different researchers provided rigorous mathematical analysis from different perspectives, and on that basis, improved the method.

\subsection{Recent developments.}

3.2.1. Simplification of the renormalization group method. To better understand the RG method in the SPT context, Ziane [146] contributed to the mathematical analysis of this technique through the study of differential equations modeling the dynamics of autonomous nonlinear systems. Not only did he introduce how to estimate the difference between the asymptotic solution and the original solution, but he also established a connection between the RG method and first-order normal form (NF) theory. To see how this was done consider the following vector equation:

$$
\begin{aligned}
& \frac{\mathrm{d} \mathbf{u}}{\mathrm{d} \tau}+\frac{1}{\varepsilon} \mathbf{A} \mathbf{u}=\mathbf{F}(\mathbf{u}), \\
& \left.\mathbf{u}\right|_{\tau=0}=\mathbf{u}_{0},
\end{aligned}
$$

where $0<\varepsilon \ll 1$, $\mathbf{A}$ is a complex diagonal matrix, and $\mathbf{F}$ is a polynomial

nonlinear term. Apply the time scale transformation $t=\frac{\tau}{\varepsilon}$, and Eq. (3.3) becomes the following equation:

$$
\begin{aligned}
& \frac{\mathrm{d} \mathbf{u}}{\mathrm{d} t}+\mathbf{A} \mathbf{u}=\varepsilon \mathbf{F}(\mathbf{u}), \\
& \left.\mathbf{u}\right|_{t=0}=\mathbf{u}_{0} .
\end{aligned}
$$

Through the renormalization analysis in Section 3.1, we obtain the RG equation:

$$
\begin{gathered}
\frac{\mathrm{d} \mathbf{V}}{\mathrm{d} \tau}=\mathbf{R}(\mathbf{V}), \\
\left.V\right|_{\tau=0}=v_{0},
\end{gathered}
$$

where $\mathbf{R}(\cdot)$ is the resonant part of the polynomial $\mathbf{F}(\cdot)$ relative to the operator $\mathbf{A}$. The final uniformly valid formal solution is:

$$
\mathbf{u}(\tau)=e^{-\frac{\tau}{\varepsilon} \mathbf{A}} \mathbf{V}(\tau)+\varepsilon \mathbf{G}(\mathbf{V}(\tau))+O\left(\varepsilon^{2}\right),
$$

where the form of function $\mathbf{G}(\cdot)$ is related to the specific form of the nonlinear function $\mathbf{F}(\cdot)$. By applying the mathematical analysis this asymptotic validity is up to $O(\varepsilon)$.

In summary, the RG method applied to SPT can be expressed in three steps:

(1) Write a naive perturbation expansion solution of the equation, which always contains secular terms.

(2) Introduce a free parameter to remove the secular terms. 
(3) Derive the RG equation by using the fact that the approximate solution of the perturbed problem is independent of the free parameter.

\subsubsection{Simplification and comparison to normal form theory.}

DeVille et al. 32, 33 not only examined the mathematical basis of the RG method but derived a simplified algorithm for this method up to $O\left(\varepsilon^{2}\right)$. In addition, the crucial step of the RG method is a near-identity change of coordinates. It is equivalent to the NF theory for systems with autonomous/ non-autonomous perturbations to second-order and higher-order systems.

Consider the following weakly nonlinear autonomous ordinary differential equation (ODE):

$$
\frac{\mathrm{d} \mathbf{x}}{\mathrm{d} t}=\mathbf{A x}+\varepsilon \mathbf{f}(\mathbf{x})
$$

and weakly nonlinear non-autonomous ODE:

$$
\frac{\mathrm{d} \mathbf{x}}{\mathrm{d} t}=\mathbf{A x}+\varepsilon \mathbf{f}(\mathbf{x}, t)
$$

where $0<\varepsilon \ll 1$, $\mathbf{A}$ is a complex diagonal matrix with purely imaginary eigenvalues, and $\mathbf{f}(\mathbf{x}), \mathbf{f}(\mathbf{x}, t)$ are both smooth functions.

Based on the previous studies, a simplified version that highlights the mathematical underpinnings of the RG method can be shown as the following steps:

(1) Derive a naive perturbation expansion for the solution of the given differential equation with an arbitrary initial time $t_{0}$ and initial condition $\mathbf{x}\left(t_{0}\right)$.

(2) Renormalize the initial condition by absorbing those terms in the naive expansion which are time-independent and bounded into $\mathbf{x}\left(t_{0}\right)$.

(3) Apply the RG condition:

$$
\frac{\mathrm{d} \mathbf{x}}{\mathrm{d} t_{0}}=0
$$

By showing the relationship between the RG method and the NF theory, the diagram can be summarized as the following form:

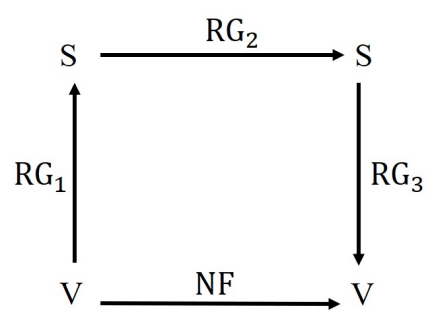


where $\mathrm{V}$ denotes the space of vector fields, $\mathrm{S}$ denotes the space of truncated asymptotic expansion. $\mathrm{RG}_{1}, \mathrm{RG}_{2}$ and $\mathrm{RG}_{3}$ represent the above three steps, respectively. NF denotes the change of coordinates central to the NF method.

The essential reductive step in the RG method is the change of coordinates known as $\mathrm{RG}_{2}$. This coordinate change is near-identity on time scales of $O(1 / \varepsilon)$. It absorbs the non-resonant terms from the asymptotic expansion into the initial condition, which is similar to the coordinate change involving the dependent variable in the NF theory. These two methods are used together to remove the non-resonance term. Holzer et al. [57 developed this theory and used three examples to illustrate that the RG method can well handle the perturbation problem with logarithmic switching terms.

The advantages of the RG method over the NF method are that the secular terms are easier to identify, and the form of the near-identity coordinate change is not necessarily known in advance so that the RG method is quite general.

3.2.3. The geometric meaning. In geometry, the envelope of a family of curves in the plane is a curve that is tangent to each member of the family in at least one point, and these points of tangency together form the entire envelope. Let $\left\{C_{\tau}\right\}_{\tau}$ denote a family of curves indexed by $\tau$ in the $x-y$ plane, where $C_{\tau}$ is implicitly given by the equation

$$
F(x, y, \tau)=0 \text {. }
$$

The envelope of $\left\{C_{\tau}\right\}_{\tau}$ is defined as the set of all $(x, y) \in \mathbb{R}^{2}$ satisfying the following equations

$$
\begin{aligned}
& F(x, y, \tau)=0, \\
& \frac{\partial F}{\partial \tau}(x, y, \tau)=0,
\end{aligned}
$$

for some values of the parameter $\tau$. For more details, see [1, 15].

To establish the geometric meaning of the solution of the differential equation Kunihiro [67, 68] showed that the RG equation can be interpreted as an envelope equation, which is known as the envelope of the family of curves representing naive approximations. Kunihiro et al. [36] also derived reduced equations for evolution on attracting slow manifolds and approximate center manifolds in perturbed ODEs.

3.2.4. Invariant conditions. Independently and simultaneously with the works of Chen et al., Woodruff [137, 138] proposed an invariance condition that shares many features with the RG method. It was used to treat multiscale perturbation problems, that is, by introducing implicit invariance in the expansion process, a uniformly valid solution can be constructed. It 
is the invariance condition that is analogous to the $\mathrm{RG}$ condition, and Woodruff's method yields the same types of results as the RG method.

3.2.5. New average ansatz. Mudavanhu and O'Malley Jr [79 analyzed the asymptotic solution of a particular class of initial value vector system:

$$
\dot{\mathbf{x}}=\mathbf{M x}+\varepsilon \mathbf{N}(\mathbf{x}, t, \varepsilon),
$$

where $\mathbf{M}$ is a diagonal matrix with only imaginary eigenvalues and $\mathbf{N}(\mathbf{x}, t, \varepsilon)$ is a smooth matrix function concerning all three variables and is $p$-periodic in $t$. This system is subject to small-amplitude time-periodic perturbations.

Change the dynamic variables as follows:

$$
\mathbf{z}(t)=e^{-\mathbf{M} t} \mathbf{x}(t),
$$

that will take the transformed system to the standard form:

$$
\dot{\mathbf{z}}(t)=\varepsilon \mathbf{f}(\mathbf{z}, t, \varepsilon),
$$

where the new function is defined by:

$$
\mathbf{f}(\mathbf{z}, t, \varepsilon)=e^{-\mathbf{M} t} \mathbf{N}\left(e^{\mathbf{M} t} \mathbf{z}, t, \varepsilon\right) .
$$

To solve this equation perturbatively introduce the regular power series expansion:

$$
\mathbf{z}(t)=\mathbf{z}_{0}(t)+\varepsilon \mathbf{z}_{1}(t)+\varepsilon^{2} \mathbf{z}_{2}(t)+\cdots,
$$

where $\mathbf{z}_{i}(t)(i=0,1,2, \cdots)$ satisfy the following sequence of linear initial value problems:

$$
\begin{aligned}
& \varepsilon^{0}: \dot{\mathbf{z}}_{0}(t)=0, \mathbf{z}_{0}(0)=\mathbf{x}(0) \\
& \varepsilon^{1}: \dot{\mathbf{z}}_{1}(t)=\mathbf{f}\left(\mathbf{z}_{0}(t), t, 0\right), \mathbf{z}_{1}(0)=0 \\
& \varepsilon^{2}: \dot{\mathbf{z}}_{2}(t)=\mathbf{f}_{z}\left(\mathbf{z}_{0}(t), t, 0\right) \mathbf{z}_{1}(t)+\mathbf{f}_{\varepsilon}\left(\mathbf{z}_{0}(t), t, 0\right), \mathbf{z}_{2}(0)=0 \\
& \quad \cdots
\end{aligned}
$$

and integrating successively, we can obtain the hierarchy of solutions:

$$
\begin{aligned}
& \mathbf{z}_{0}(t)=\mathbf{x}(0) \\
& \mathbf{z}_{1}(t)=\int_{0}^{t} \mathbf{f}(\mathbf{x}(0), s, 0) \mathrm{d} s \\
& \mathbf{z}_{2}(t)=\int_{0}^{t}\left[\mathbf{f}_{z}(\mathbf{x}(0), s, 0) \mathbf{z}_{1}(s)+\mathbf{f}_{\varepsilon}(\mathbf{x}(0), s, 0)\right] \mathrm{d} s, \\
& \ldots
\end{aligned}
$$

Partition the function $\mathbf{f}(\mathbf{x}(0), t, 0)$ into an average part and a fluctuating zero-average part:

$$
\mathbf{f}(\mathbf{x}(0), t, 0)=\langle\mathbf{f}(\mathbf{x}(0), t, 0)\rangle+\{\mathbf{f}(\mathbf{x}(0), t, 0)\},
$$


where the non-zero average is given by:

$$
\langle\mathbf{f}(\mathbf{x}(0), t, 0)\rangle \equiv \frac{1}{p} \int_{0}^{p} \mathbf{f}(\mathbf{x}(0), s, 0) \mathrm{d} s,
$$

which is the new ansatz when considering the simple resonance problem.

By using this ansatz, $\mathbf{z}_{1}(t)$ can be separated into a secular part and a bounded secular-free part

$$
\mathbf{z}_{1}(t)=\boldsymbol{a}_{0} t+\mathbf{Z}_{1}(t)
$$

where the secular part is $\boldsymbol{a}_{0} t$, the average is:

$$
\boldsymbol{a}_{0}=\langle\mathbf{f}(\mathbf{x}(0), t, 0)\rangle
$$

and the bounded secular-free part is:

$$
\mathbf{Z}_{1}(t)=\int_{0}^{t}\{\mathbf{f}(\mathbf{x}(0), s, 0)\} \mathrm{d} s .
$$

Using the similar treatment, $\mathbf{z}_{2}(t)$ possesses the same form. Finally, apply the multi-scale in a slow time $\tau=\varepsilon t$, the secular term in the direct expansion solution can be separated and removed.

3.2.6. Lie-group approach. Goto et al. 51 constructed a representation of the Lie group from the renormalization transformation in several examples and derived an asymptotic expression for a generator of the Lie group. This novel perspective points out that the RG method can be understood as the procedure by which to obtain an asymptotic expression for a generator of the renormalization transformation based on the Lie group.

This Lie-group approach provides the following simple recipe for obtaining the asymptotic form of the RG equation from an ODE to a partial differential equation (PDE):

(1) By utilizing the naive perturbation calculations, obtain a secular series solution of the perturbed equation.

(2) Find the renormalized integral constant to eliminate all the secular terms in the perturbation solution and give the renormalized transformation.

(3) Rewrite the renormalization transformation by performing an arbitrary shift operation on the independent variable: $t \rightarrow t+\tau$ and obtain a representation of the Lie group underlying the renormalization transformation.

(4) By differentiating the representation of the Lie group to arbitrary $\tau$, obtain an asymptotic expression of the generator, thereby generating an asymptotic RG equation.

\subsection{Nine applications of the renormalization group method.}


3.3.1. Stochastic differential equations. In real physical systems, uncertainties that are difficult to explain using basic models occur due to measurement errors, unresolved scales/interactions, and numerical inaccuracies. It is desirable to quantify the robustness of models, especially those involving singular perturbations, in the presence of these uncertainties. Therefore, it is natural to combine small amplitude white noise driven disturbances into existing singular models.

Blömker et al. 13 applied the RG method to the study of the stochastic driven system, which is the stochastic two-dimensional Navier-Stokes equation with additive Gaussian noise and periodic boundary conditions.

Glatt-Holtz and Ziane [4] studied a class of stochastic differential equations:

$$
\mathrm{d} \mathbf{X}+\frac{1}{\varepsilon} \mathbf{A} \mathbf{X} \mathrm{d} \tau=\mathbf{F}(\mathbf{X}) \mathrm{d} \tau+\varepsilon^{m} \mathbf{G}(\tau, \tau / \varepsilon) \mathrm{d} \mathbf{W},
$$

where $\mathbf{A}$ is a linear operator that assume to be symmetric positive semidefinite or antisymmetric; $\mathbf{F}(\cdot)$ is a polynomial function; $\mathbf{G}(\cdot, \cdot)$ takes values in $\mathbb{M}^{n \times n}$ and is bounded in the Frobenius norm sense; $m$ is a constant; $\mathrm{d} \mathbf{W}$ is the white noise process. By using the RG method on Eq. (3.4) we obtain the following RG equation:

$$
\mathrm{d} \mathbf{V}=\mathbf{R}(\mathbf{V}) \mathrm{d} \tau+\varepsilon^{m} \mathbf{H}(\tau, \tau / \varepsilon) \mathrm{d} \mathbf{W},
$$

and the uniformly valid formal asymptotic solution:

$$
\mathbf{X}=e^{-\mathbf{A} \tau / \varepsilon} \mathbf{V}
$$

In the sense of probability the solution is valid to the order of $O(1)$ through a strict proof.

3.3.2. Fractional stochastic differential equations. Brownian motion is the label for the motion of those particles driven by stochastic forces due to coupling to the random environment. This process is an independent incremental continuous random process with a normal PDF. Fractional Brownian motion (FBM), compared with classical Brownian motion, is one of the well-developed stochastic processes with strong long-range correlations in space and time. These stochastic processes, such as FBM and Lévy flights [38], are often used to describe phenomena with anomalous diffusive behavior and extended memory processes in the field of economics and finance. Guo et al. [54] studied a class of stochastic differential equations driven by FBM with Hurst parameter $H \in\left(\frac{1}{2}, 1\right)$ :

$$
\begin{aligned}
& \mathrm{d} \mathbf{X}(t)+\mathbf{A} \mathbf{X}(t) \mathrm{d} t=\varepsilon \mathbf{F}(\mathbf{X}(t)) \mathrm{d} t+\varepsilon^{m+H} \mathbf{G}(\varepsilon t, t) \mathrm{d} \mathbf{B}_{t}^{H}, \\
& \mathbf{X}(0)=\mathbf{X}_{0},
\end{aligned}
$$

where $\mathbf{A}$ is a linear operator that is assumed to be symmetric positive semidefinite or antisymmetric; $\mathbf{F}(\cdot)$ is the polynomial function; $\mathbf{G}(\cdot, \cdot)$ takes 
values in $\mathbb{M}^{n \times n}$ and bounded in the Frobenius norm sense; $m$ is a constant; $\mathbf{B}_{\tau}^{H}$ is the $n$-dimensional FBM, defined as the stochastic integral, for $t \geqslant 0$ :

$$
\begin{aligned}
\mathbf{B}_{t}^{H}= & \frac{1}{\Gamma(H+1 / 2)}\left\{\int_{-\infty}^{0}\left[(t-s)^{H-1 / 2}-(-s)^{H-1 / 2}\right] \mathrm{d} \mathbf{W}(s)\right. \\
& \left.+\int_{0}^{t}(t-s)^{H-1 / 2} \mathrm{~d} \mathbf{W}(s)\right\},
\end{aligned}
$$

where $\mathrm{d} \mathbf{W}(s)$ denotes an $n$-dimensional Wiener process defined on $(-\infty, \infty)$. By rigorous analysis, uniformly effective asymptotic solutions to the order of $O(1)$ are obtained. This was the first attempt to apply the RG method to fractional stochastic differential equations.

3.3.3. Hamiltonian systems. Yamaguchi and Nambu [139] discussed the relation between integrability of the original systems and the symplectic properties of the RG equations in Cartesian coordinates. Consider the following Hamiltonian systems:

$$
\begin{aligned}
H\left(q_{1}, q_{2}, p_{1}, p_{2}\right) & =H_{0}\left(q_{1}, q_{2}, p_{1}, p_{2}\right)+\varepsilon V_{1}\left(q_{1}, q_{2}\right), \\
H_{0}\left(q_{1}, q_{2}, p_{1}, p_{2}\right) & =\frac{1}{2}\left(p_{1}^{2}+p_{2}^{2}+q_{1}^{2}+q_{2}^{2}\right),
\end{aligned}
$$

where $V_{1}\left(q_{1}, q_{2}\right)$ is a homogeneous cubic or quartic function of the two canonical displacement variables $q_{1}, q_{2}$ and the perturbation parameter is quite small $|\varepsilon| \ll 1$.

The conclusion is that the original Hamiltonian systems and the RG equations are integrable if the $\mathrm{RG}$ equations are Hamiltonian systems up to the second leading-order of the small parameter. They [140] concluded that the result obtained from the $\mathrm{RG}$ method equivalent to those obtained by canonical Hamiltonian perturbation theory, up to $O\left(\varepsilon^{2}\right)$.

3.3.4. Vector field approximation on manifolds. By assuming the secular term in the RG method satisfy Krylov-Bogoliubov-Mitropolsky (KBM) condition, Chiba 26] studied the following types of ODEs:

$$
\dot{\mathbf{x}}=\mathbf{f}(t, \mathbf{x})+\varepsilon \mathbf{g}(t, \mathbf{x}),
$$

in terms of the simple perturbation series:

$$
\dot{\mathbf{x}}=\mathbf{F} \mathbf{x}+\varepsilon \mathbf{g}_{1}(t, \mathbf{x})+\varepsilon^{2} \mathbf{g}_{2}(t, \mathbf{x})+\cdots+\varepsilon^{m} \mathbf{g}_{m}(t, \mathbf{x}),
$$

where $\mathbf{x} \in \mathbb{R}^{n} ; \mathbf{f}(t, \mathbf{x})$ and $\mathbf{g}(t, \mathbf{x})$ are $C^{4}$ and $C^{3}$ time-dependent vector field functions; $\mathbf{F}$ is a diagonalizable $n \times n$ matrix with pure imaginary eigenvalues; and $\mathbf{g}_{1}(t, \mathbf{x}), \cdots, \mathbf{g}_{m}(t, \mathbf{x})$ are $C^{\infty}$ vector field functions, which are polynomials in $\mathbf{x}$ and periodic in $t$.

Furthermore, he concluded that the vector field is defined by the approximate solutions obtained using the RG method, which is close to the 
original vector field in the $C^{1}$ topology. Furthermore, he considered the following ODEs

$$
\dot{\mathbf{x}}=\mathbf{F} \mathbf{x}+\varepsilon \mathbf{g}(\mathbf{x}),
$$

along with the perturbation series:

$$
\dot{\mathbf{x}}=\mathbf{F} \mathbf{x}+\varepsilon \mathbf{g}_{1}(\mathbf{x})+\cdots+\varepsilon^{m} \mathbf{g}_{m}(\mathbf{x}),
$$

where as before $\mathbf{F}$ is a diagonalizable $n \times n$ constant matrix with pure imaginary eigenvalues and $\mathbf{g}(\mathbf{x}), \mathbf{g}_{1}(\mathbf{x}), \cdots, \mathbf{g}_{m}(\mathbf{x})$ are vector polynomials on $\mathbb{R}^{n}$.

According to Fenichel theory, some topological properties of the original vector field can be obtained, that means, if the approximate vector field has normally hyperbolic invariant manifolds, then the original vector field is also expected to have the same invariant manifolds.

Chiba [27] studied a more general class of singular perturbation problems:

$$
\dot{\mathbf{x}}=\varepsilon \mathbf{g}(\mathbf{x}, t, \varepsilon),
$$

where $\mathbf{x} \in U, U$ is an open set in $\mathbb{C}^{n}$ and $\mathbf{g}(\mathbf{x}, t, \varepsilon)$ is a vector field and is almost-periodic in $t$. The RG method can also lead to the existence of approximate invariant manifolds, the inheritance of symmetries from the original equation to the RG equation. Li and Shi 69 extended the condition to a more general situation by using the RG method to study the case where $\mathbf{g}(\mathbf{x}, t, \varepsilon)$ is quasi-periodic in $t$ and they obtained a number of rigorous approximate results.

3.3.5. Energy conservation and dissipation. Based on the above averaging ansatz, Temam and Wirosoetisno [117] considered the approximate solution to a class of ODEs that were initiated from a fluid dynamics background:

$$
\begin{aligned}
& \frac{\mathrm{d} \mathbf{u}}{\mathrm{d} t}+\frac{1}{\varepsilon} \mathbf{L u}+\mathbf{A} \mathbf{u}+\mathbf{B}(\mathbf{u})=\mathbf{f}, \\
& \mathbf{u}(0)=\mathbf{u}_{0},
\end{aligned}
$$

where $\mathbf{u} \in \mathbb{R}^{d}, \mathbf{L}$ is a real antisymmetric matrix, $\mathbf{A}$ is a positive definite matrix, $\mathbf{f}=\mathbf{f}(t)$ is a function with the bounded infinite norm, and $\mathbf{B}(\mathbf{u})$ is referred to as the nonlinear part.

Two conclusions that follow from their analysis are:

(1) When using the RG method, the leading-order approximation model obtained has the same energy conservation and dissipation properties as the original model, but the high-order approximations are no longer maintained.

(2) The higher-order solutions are still bounded, and like the solution of the original equation, they eventually falls 
into an absorbing set associated with a sufficiently small parameter $\varepsilon$.

3.3.6. Periodic orbit problems. Based on the RG method, Bhattacharjee et al. [11 identified and classified the periodic solutions - limit cycle behavior or orbiting around a center - in various types of two-dimensional nonlinear dynamical systems. Subsequently, Palit and Datta 88] improved the RG analysis by invoking the concept of nonlinear time. They studied the Rayleigh and Van der Pol equations and for the amplitude $a(\varepsilon)$ of the limit cycle of the two oscillators, derived the effective approximate formula as a function of the nonlinear parameter $\varepsilon$. Additionally, they proposed good approximate plots of the limit cycle of the oscillator.

3.3.7. Boundary layer problems. For a class of singular perturbation problems, the domain can be divided into two or more subdomains. In one of these (usually the largest) subdomains, the solution can be obtained as an asymptotic series through regular perturbation theory. However, this approximate solution is inaccurate in other subdomains. If these subdomains are on the boundary of the domain, it is called the boundary layer. If it is in the middle of the domain it is called the inner layer.

Consider the following boundary layer problem:

$$
\begin{aligned}
& \varepsilon \ddot{\mathbf{y}}+\mathbf{p}(x) \dot{\mathbf{y}}+\mathbf{q}(x) \mathbf{y}=0, \quad 0 \leqslant x \leqslant 1 \\
& \mathbf{y}(0)=\boldsymbol{\alpha}, \quad \mathbf{y}(1)=\boldsymbol{\beta},
\end{aligned}
$$

where $\mathbf{p}(x)$ and $\mathbf{q}(x)$ are $C^{r}(r \geq 2)$ functions, and $\mathbf{p}(x)>0$ on the interval $[0,1]$. When dealing with the above boundary layer problem, Zhou et al. [145] recently showed that the RG method has the same efficiency as the classical matching asymptotic expansion method to order $O(\varepsilon)$. The latter can be seen as an extension of Kirkinis's work [65].

3.3.8. Fast-slow systems. Consider the following fast-slow system motivated by biological applications:

$$
\begin{aligned}
\frac{\mathrm{d} \mathbf{u}_{\varepsilon}}{\mathrm{d} t} & =\mathbf{f}\left(\mathbf{u}_{\varepsilon}, v_{\varepsilon}\right), t>0, \mathbf{u}_{\varepsilon}(0)=\mathbf{u}^{0}, \\
\varepsilon \frac{\mathrm{d} \mathbf{v}_{\varepsilon}}{\mathrm{d} t} & =-\alpha \mathbf{v}_{\varepsilon}+\Phi\left(\mathbf{u}_{\varepsilon}, \mathbf{v}_{\varepsilon}\right), t>0, \mathbf{v}_{\varepsilon}(0)=\mathbf{v}^{0},
\end{aligned}
$$

where $\mathbf{f}$ and $\boldsymbol{\Phi}$ are defined on $\mathbb{R}^{m+1}$ and take on values respectively in $\mathbb{R}^{m}$ and $\mathbb{R}^{1}$ with $m \geqslant 1$.

By using the RG method, Marciniak-Czochra et al. [73] derived the $O\left(\varepsilon^{2}\right)$ approximation of Eq. (3.5) and Eq. (3.6), which only relates to the variables of the slow equations given in Eq. (3.5). Furthermore, the proof of the $\varepsilon^{2}$-order error estimate over an infinite interval is provided. The 
contribution indicates that the RG method is more straightforward than the boundary layer calculation in the classic method of Vasil'eva [123.

3.3.9. Partial differential equations. The RG method is also rigorously applied to some PDEs on bounded intervals, such as the Navier-Stokes equations [75], a slightly compressible fluid equation, the Swift-Hohenberg equation [23, 76, 100, Maxwell's wave equation [28, as well as the primitive equations of the atmosphere and the ocean [93].

Salem [99] studied the quadratic nonlinear Schrödinger equation:

$$
i \partial_{t} \phi=-\Delta \phi-\varepsilon \phi^{2},
$$

where $\Delta$ is the spatial Laplacian operator in $\mathbb{R}^{3}$. He concluded that the approximate solution holds for a long time $\left(t_{0} \leqslant t \leqslant t_{0}+\delta \frac{|\log \varepsilon|}{e \phi_{0} \varepsilon}, \delta \in(0,1)\right)$. Moreover, the approximate solution is compared with the true solution in space $L^{2}\left(\mathbb{R}^{3}\right)$.

Pocovnicu 94 obtained an approximate solution of the nonlinear halfwave equation which is the Schrödinger equation in a non-dispersive situation:

$$
i \partial_{t} \phi-|D| \phi=|\phi|^{2} \phi
$$

where $D=-i \partial_{x}$. The approximation holds for a long time $(0 \leqslant t \leqslant$ $\left.C \varepsilon^{-2}\left[\log \left(1 / \varepsilon^{\delta}\right)\right]^{1-2 \alpha}, 0 \leqslant \alpha \leqslant 1 / 2, \delta \in(0,1)\right)$, and the approximate solution also compares favorably with the true solution in space $H_{+}^{s}(\mathbb{R})$, for $s>1 / 2$.

An approximation was obtained for the solution of the quadratic dispersive equations by Wang [124]:

$$
\begin{aligned}
& i \partial_{t} u+\Delta u=-\varepsilon v^{2}, u(0, x)=0, \\
& i \partial_{t} v+\Delta v=0, v(0, x)=v^{*}(x) .
\end{aligned}
$$

The approximation holds for a long time $\left(0 \leqslant t \leqslant \frac{\delta|\log \varepsilon|}{e \varepsilon \alpha}, \delta \in(0,1)\right)$, and the approximation solution also compared with the true solution in space $L^{p}(\mathbb{R})$, for $p \in[2, \infty]$.

\section{Connecting renormalization group and fractional calculus theories}

We have reviewed, all too briefly, the origin and developments of the RG method in different fields, and now turn our attention to determining the connections between RG and FC theory. The essential difference between traditional calculus and FC lies in the definition of the classical operations of differentiation and integration. Classically the derivative is a "local" operator, it is uniquely determined at the point $x$, where in dynamics that point denotes either space or time and along with the definition of the limit 
forms the basis of Newtonian mechanics. Newton's explicit assumption that space is homogeneous and time is isotropic along with definitions of the ordinary calculus are the mathematical foundations of classical physics.

The fractional derivative has "non-local" or "integral" properties in that a fractional derivative in time has memory and a fractional derivative in space depends on the behavior of the process arbitrarily far away. Consequently, the fractional derivative of $f(x)$ depends on all its values, not just the value of the function in a small neighborhood of $x$. Just as in geometry having more than one way to generalize beyond the form of Euclid, there are multiple ways to generalize the definitions of the derivative and integral operators. For example, different formal expressions for fractional derivatives are given in Appendix $\mathrm{A}$.

At present, although the existing literature attempts to combine RG and FC from different disciplines, there is a lack of in-depth and essential research on the relationship between these two theories.

Douglas [35] studied the surface-interacting polymers model and obtained the exact solution of the partition function using the FC. Moreover, he explained the basic relationship between the $\mathrm{RG}$ scaling functions and the exact scaling functions. This is the first research paper combining the $\mathrm{RG}$ and FC theories that we could find.

4.1. Fractional Brownian motion. Quan [96 studied the connection between FBM and the RG in statistical physics, and determined statistical, geometric, and fractal properties of complex phenomena. The RG method is a powerful tool for qualitatively studying physical phenomena as well as pure mathematical problems with scaling properties. Meanwhile, some complex systems with IPL properties can also be studied using the FC theory.

As shown in Fig. 4, when a beam of white light enters a dispersive prism, the prism decomposes the white light into its constituent spectral colors (the colors of the rainbow). Complex systems are equivalent to such a beam of white light, which can be decomposed into its various spectra (observations) through a dispersive prism system. These observations are typical representations of complex systems: scale-free, self-similarity, longrange dependence, and long-term memory.

So, what is this prism system (or lens) that connects complexity with its macroscopic observations? Through discussions in the previous sections, we tried to explore the essence of complex systems through phenomena from different angles and found that the "lens" is the IPL or scaling laws. Through this "lens", RG and FC become the common ground method to reveal the essence of complexity. Therefore, it should emphasize that these 


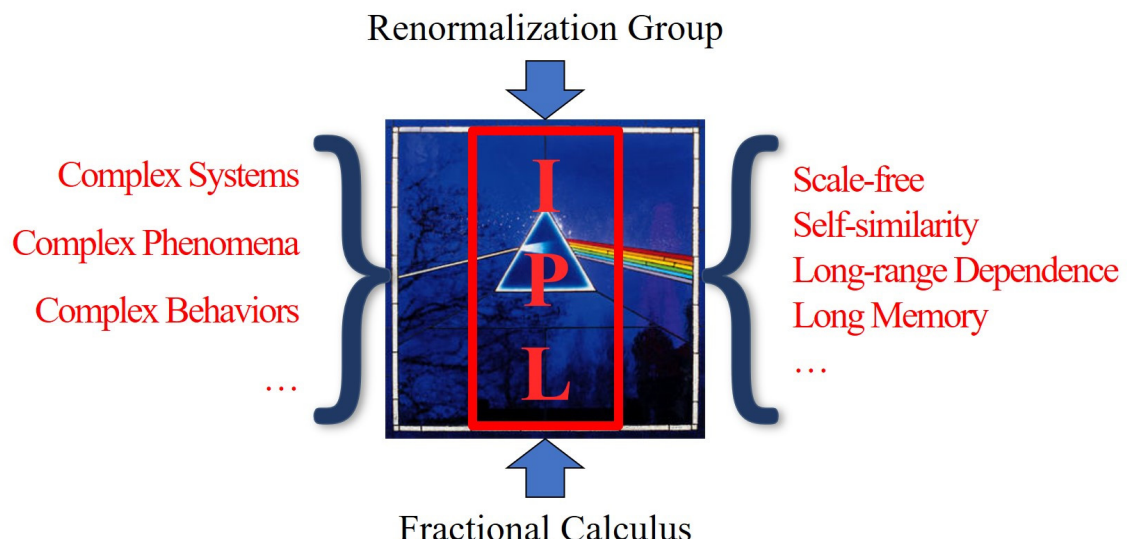

FIgURE 4. The connection between renormalization group and fractional calculus.

two research areas are by nature connected. This connection is the IPL and scaling laws, both are essential for studying complex systems.

This point can be made directly by way of example wherein we consider a random walk process determined by a fractal function and called the Weierstrass random walk (WRW) [59] for reasons that will become obvious in due course. Consider the discrete probability described by the stepping PDF for the WRW on a one-dimensional lattice with sites indexed by $q$ :

$$
P(q)=\frac{a-1}{2 a} \sum_{n=0}^{\infty} \frac{1}{a^{n}}\left[\delta_{q, b^{n}}+\delta_{q,-b^{n}}\right],
$$

where $a$ and $b$ are dimensionless constants greater than one. $\delta_{i j}$ is the Kronecker delta function: $\delta_{i j}=1$ for $i=j$ and $\delta_{i j}=0$ for $i \neq j$. We follow the analysis of this discrete process given by West and Grigolini [130. The first property of note is that the second moment of this diffusive process is:

$$
\left\langle q^{2}\right\rangle=\frac{a-1}{a} \sum_{n=0}^{\infty}\left(\frac{b^{2}}{a}\right)^{n},
$$

which diverges for $b^{2}>a$ since the series is infinite. The lattice structure function $\widehat{P}(k)$ is the discrete Fourier transform of the PDF and consequently is the discrete version of the characteristic function:

$$
\widehat{P}(k)=\frac{a-1}{a} \sum_{n=0}^{\infty} \frac{1}{a^{n}} \cos \left[b^{n} k\right] .
$$


This series was introduced by Weierstrass in 1872 [71] to represent a process that is continuous everywhere but is nowhere differentiable. Thanks to Mandelbrot we now know that this was the first consciously constructed fractal function and the divergence of the second moment is a consequence of its non-analytic properties.

As the WRW process proceeds the set of sites visited consists of localized clumps of sites, interspersed by gaps, nested within clusters of clumps over ever-larger spatial scales. The WRW generates a hierarchy of clumps that are statistically self-similar, as suggested by Fig. 5. The parameter $a$ determines the number of subclusters within a cluster and the parameter $b$ determines the scale size between groupings.

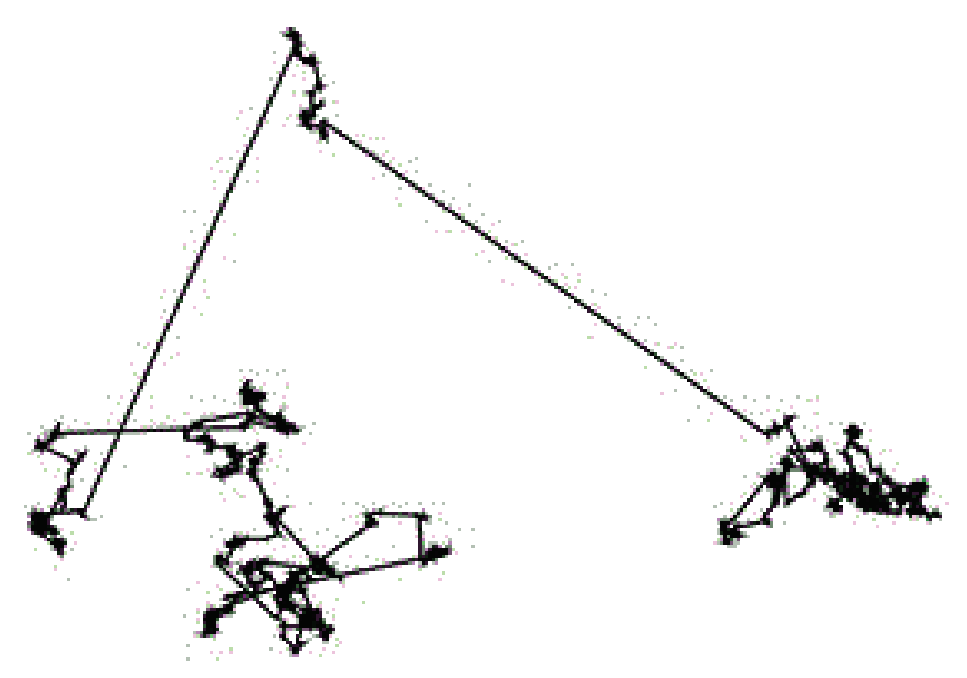

FiguRE 5. The landing sites for the WRW are depicted and the islands of clusters are radially seen.

Let us now apply the RG method to the lattice structure function and determine the scaling properties of the WRW. Scaling the argument of the lattice structure function by $b$ and reordering terms in the series yields:

$$
\widehat{P}(b k)=a \widehat{P}(k)-(a-1) \cos k .
$$

The solution to this RG equation can be separated into homogeneous and singular parts and the singular part $\widehat{P}_{s}(k)$ is obtained by solving the homogeneous scaling equation:

$$
\widehat{P}_{s}(b k)=a \widehat{P}_{s}(k) .
$$


The formal solution to this equation is given by:

$$
\widehat{P}_{s}(k)=A(k) k^{\mu}
$$

which when inserted into Eq. (4.1) yields:

$$
A(b k) b^{\mu} k^{\mu}=a A(k) k^{\mu} .
$$

The last equality implies that $A(k)$ is periodic in the logarithm of $k$ with period $\ln b, \mu=\ln a / \ln b$ and the solution can be written:

$$
\widehat{P}_{s}(k)=\sum_{n=-\infty}^{\infty} A_{n}|k|^{H_{n}},
$$

with the complex power-law index:

$$
H_{n}=\mu+i n \frac{2 \pi}{\ln b}=\frac{\ln a}{\ln b}+i n \frac{2 \pi}{\ln b} .
$$

The analytic forms of the Fourier coefficients in Eq. (4.2) are given in Hughes et al. [59].

It is possible to prove that the dominant behavior of the WRW is determined by the lowest-order term in the singular part of the solution for the lattice structure function but we do not do that here. We assume that the dominant behavior is given by the $n=0$ term in the series:

$$
\widehat{P}_{s}(k) \approx A_{0}|k|^{\mu},
$$

whose inverse Fourier transform is the IPL:

$$
P(q)=\frac{K(\mu)}{|q|^{\mu+1}},
$$

and $K(\mu)$ is a known function of $\mu$. Thus, the singular part of the WRW has an IPL stepping PDF and this behavior intuitively justifies ignoring all the other terms in the series.

We now write for the time-dependent form of the discrete PDF:

$$
P(q, n+1)=\sum_{q^{\prime}} P\left(q-q^{\prime}\right) P\left(q^{\prime}, n\right)=\sum_{q^{\prime}} \frac{K(\mu)}{\left|q-q^{\prime}\right|^{\mu+1}} P\left(q^{\prime}, n\right),
$$

where we assume that each step $n$ in WRW process occurs at equal time intervals. This equation was analyzed in 1970 by Gillis and Weiss [46], who determined that its solution is a Lévy PDF, thereby connecting the RG solution the WRW to discussion of the fractional diffusion equation given earlier. 
4.2. Fractional kinetic equations. Zaslavsky [143] studied chaotic particle kinetics generated by a class of non-integrable Hamiltonians. He derived the fractional Fokker-Planck-Kolmogorov (FFPK) equation for the $\mathrm{PDF}$ to describe the ensemble behavior of the random walk in the fractal space-time system. Using the RG method, he was able to solve the FFPK equation and concluded that the critical exponents of the anomalous kinetics represented the dynamics of the Hamiltonian. That is, when the fixed points of the RG equation exist, they lead to the relationship between the space-time fractional exponents and the space-time scale expressed in the scaling form of the PDF solution. It is a pioneering work applying RG theory to fractional-order stochastic dynamical systems.

Consider a random walk generated by a Hamiltonian system whose dynamics are chaotic. The names Kolmogorov [66], Arnold [7] and Moser [78] are associated with laying out the boundaries of classical mechanics and as West and Grigolini 130, explain, KAM theory describes how the continuous trajectories of particles determined by a Hamiltonian break up into a chaotic sea of randomly disconnected points. This kind of "strange kinetics" produces the FFPK equations to describe the evolution of the PDFs in phase space. Schematically we write the FFPK equation for an ensemble of chaotic trajectories as:

$$
\delta_{t} P(l, t)=\delta_{l} P(l, t)
$$

where $\delta_{t}$ is the infinitesimal time-variation of the PDF and $\delta_{l}$ is the corresponding variational quantity in space.

The application of the RG transform $\mathcal{R}$ to this equation $n$-times yields for an ensemble of chaotic trajectories:

$$
\mathcal{R}^{n}\left[\delta_{t} P(l, t)\right]=\mathcal{R}^{n}\left[\delta_{l} P(l, t)\right],
$$

where on the left we obtain by scaling the time increment $\Delta t^{\alpha}$ :

$$
\mathcal{R}^{n}\left[\Delta t^{\alpha}\right]=\lambda_{T}^{n \alpha} \Delta t^{\alpha},
$$

and on the right we obtain by scaling the space increment $\Delta l^{\beta}$ :

$$
\mathcal{R}^{n}\left[\Delta l^{\beta}\right]=\lambda_{L}^{n \beta} \Delta t^{\beta} .
$$

Thus, in the simplest case we can write:

$$
\lambda_{T}^{n \alpha} \partial_{t}^{\alpha}[P(l, t)]=\lambda_{L}^{n \beta} K_{\alpha, \beta} \partial_{|l|}^{\beta}[P(l, t)],
$$

which diverges as:

$$
\lim _{n \rightarrow \infty}\left(\frac{\lambda_{L}^{\beta}}{\lambda_{T}^{\alpha}}\right)^{n}=\left\{\begin{array}{cl}
0, & \text { if } \lambda_{L}^{\beta}<\lambda_{T}^{\alpha} \\
\infty, & \text { if } \lambda_{L}^{\beta}>\lambda_{T}^{\alpha}
\end{array}\right.
$$

unless: 


$$
\lambda_{L}^{\beta}=\lambda_{T}^{\alpha} .
$$

Eq. (4.3) defines a nontrivial fixed point of the $R G$ relation and yields:

$$
\mu=\alpha / \beta=\ln \lambda_{L} / \ln \lambda_{T},
$$

thereby yielding the fixed point kinetic equation:

$$
\partial_{t}^{\alpha}[P(l, t)]=K_{\mu} \partial_{|l|}^{\beta}[P(l, t)] .
$$

The solution to FFPK equation is obtained by interpreting the fractional time derivative in the Caputo sense and taking the Fourier-Laplace transform of the equation from which after some algebra we obtain the generalization of Eq. (1.4):

$$
P(x, t ; \alpha, \beta)=\int_{-\infty}^{\infty} e^{i k x} e^{-K_{\mu} t^{\alpha}|k|^{\beta}} \frac{\mathrm{d} k}{2 \pi} .
$$

After rescaling and some algebra we can rewrite this equation as:

$$
P(x, t ; \alpha, \beta)=\frac{1}{t^{\mu}} P\left(\frac{x}{t^{\mu}} ; \alpha, \beta\right),
$$

thereby providing a generic form for the solution to the fixed point FFPK equation. Note that this is precisely the form of Eq. (2.2) discussed earlier.

\section{Conclusions and outlook}

This paper presents a brief history of the origin, developments, and some applications of the RG method in the physical sciences and in SPT. In addition, when dealing with complex systems, the connection between $\mathrm{RG}$ and FC is revealed and explained. This connection is tied to the form of scaling and IPLs, both of which are essential to the study of complex systems.

In this connected framework, $\mathrm{RG}$ and $\mathrm{FC}$ each entail a potentially new world view. A new way of thinking that diverges from the classical Newtonian view in unexpected ways. Therefore, RG and FC have become the strategies to address the complexity related issues of the future. The following are some suggestions for future research.

(1) Prediction and analysis of complex phenomena are the focus of future research. Therefore, it is crucial to study the dynamics of the whole system at equilibrium and in out-of-equilibrium states both theoretically and experimentally, in the tradition of such variations as done on the Kondo model [135]. 
However, the known numerical methods, such as the numerical renormalization group [16], are limited to dealing with the problem of longterm evolution. Therefore, it is desirable to develop a systematic theoretical approach to study the physical properties of equilibrium and out-ofequilibrium states in different models.

(2) When using the RG method to deal with the singular perturbation problems, a more general form of the secular term is needed instead of using only KBM condition or the average ansatz.

In the field of SPT secular terms may take many forms. However, people usually assume that this term is based on the KBM condition or the average ansatz. These assumptions have some limitations, such as dealing with the following problems [70]:

$$
\dot{x}(t)=\varepsilon\left(1-x^{2}(t)\right)
$$

or

$$
\dot{x}(t)=x^{2}(t)+t \sin x(t)+\varepsilon x(t) .
$$

Therefore, revising the assumptions and making the obtained RG equations with a more general form is also a problem that needs to be solved in the future.

(3) When studying models of physical, chemical, biological, social, and financial processes, it is common to use stochastic differential equations and master equations with fractional derivatives and fractal noise. The correlation between the fractional derivative and the corresponding general derivative to the asymptotic behavior can be evaluated by RG analysis of the renormalization model, in which the fractional-order dimension of the differential operator can be calculated. Since there are many definitions of fractional derivatives, it is worth exploring the renormalization properties of different fractional derivative operators.

(4) In many signal processing methods, noise is often modeled using Gaussian statistics. However, in many real-world or man-made systems, rather than being Gaussian, noise is more likely to be bursty or intermittent. The statistics of intermittent noise is described by a heavy tailed distribution, with many spikes, which is often difficult to characterize. Therefore, in fractional-order control and fractional-order signal processing, it is necessary to solve the problem of finding a suitable filter for a given input heavy tailed noise in order to produce the output noise with a desired non-heavy-tailed distribution, using Laplace or Mellin transform [87].

Applying the RG method to the processing of non-Gaussian noise is an area worthy of development. There have been some related research efforts, such as applying the RG method in the context of extreme value theory and to the generalized CLT [2]. 
(5) As shown in Section 4, the FC and fractal processes can be used to describe long-term memory processes and long-range dependent processes. However, recent empirical analysis of complex nonlinear dynamic systems raises questions about the capabilities of the constant-order fractional processes. The root cause of these problems is that a constant Hurst parameter fails to capture the multiscaling or multifractal characteristics of a fractal random process. In order to accurately analyze complex signals with self-similarity, there have been some attempts, such as introducing a multifractional process with a time-dependent local Hurst exponents known as multifractional Brownian motion [91, 105]

$$
\begin{aligned}
\mathbf{B}_{t}^{H(t)}(t)= & \frac{1}{\Gamma(H(t)+1 / 2)}\left\{\int_{-\infty}^{0}\left[(t-s)^{H(t)-1 / 2}-(-s)^{H(t)-1 / 2}\right] \mathrm{d} \mathbf{W}(s)\right. \\
& \left.+\int_{0}^{t}(t-s)^{H(t)-1 / 2} \mathrm{~d} \mathbf{W}(s)\right\}
\end{aligned}
$$

where $H(t)$ is a Hölder exponent. Therefore, the multiscale RG [30] scheme could be applied to analyze the above complex signals with multiscaling and multifractal characteristics.

(6) At present, in terms of applications, fractional-order systems we believe will have more comprehensive applications than integer-order systems. Therefore, the application of the RG method to the fractional-order system will also be an exciting research direction [111. For example, the RG method can be used to reveal the relationship between the FC and the properties (persistent correlation, self-similarity, and variance divergence) of fractional-order stochastic processes and anomalous diffusion 38 .

We foresee a bright future implementing the synthesized application of the RG and the FC to problems emerging from complexity from in all the disciplines of science. We anticipate that the FC the RG method will be successfully applied to a broader range of fractional-order systems, and the analysis of the properties of complex system will be made even more tractable. It is our sincere hope that the present review will be useful in studying complex dynamics with "useful consequences".

\section{Appendix A. Fractional calculus}

There are various definitions of fractional differential-integral operators, not necessarily equivalent to each other. Basic fractional derivatives include Riemann-Liouville derivative, Grünwald-Letnikov derivative, Caputo derivative and Riesz derivative. For more details and properties, see [64, 95].

The Riemann-Liouville fractional derivative is expressed as the integral with a memory kernel: 


$$
{ }^{R L} D_{t}^{\alpha} f(t)=\frac{1}{\Gamma(n-\alpha)} \frac{\mathrm{d}^{n}}{\mathrm{~d} t^{n}} \int_{a}^{t} \frac{f(\tau)}{(t-\tau)^{\alpha-n+1}} \mathrm{~d} \tau, \quad t>a,
$$

where ${ }^{R L} D_{t}^{\alpha}$ represents the Riemann-Liouville derivative operator, $f(t)$ is an arbitrary integrable function over the interval $[a, t], \alpha \in(n-1, n)$, and $\Gamma(\cdot)$ is the Gamma function.

The Grünwald-Letnikov derivative is expressed as limit of the discrete operator:

$$
{ }^{G L} D_{t}^{\alpha} f(t)=\lim _{h \rightarrow 0} h^{-\alpha} \sum_{j=0}^{\left[\frac{t-t_{0}}{h}\right]}(-1)^{j}\left(\begin{array}{l}
\alpha \\
j
\end{array}\right) f(t-j h),
$$

where ${ }^{G L} D_{t}^{\alpha}$ represents the Grünwald-Letnikov derivative operator, [.] is the approximate recurrence term for integer part of the bracketed term, and $\left(\begin{array}{c}\alpha \\ j\end{array}\right)=\frac{\Gamma(\alpha+1)}{j ! \Gamma(\alpha-j+1)}$.

The Caputo fractional derivative is expressed as the integral with a memory kernel:

$$
{ }^{C} D_{t}^{\alpha} f(t)=\frac{1}{\Gamma(n-\alpha)} \int_{a}^{t} \frac{f^{(n)}(\tau)}{(t-\tau)^{\alpha-n+1}} \mathrm{~d} \tau, \quad t>a,
$$

where ${ }^{C} D_{t}^{\alpha}$ represents the Caputo derivative operator, $n$ is an integer, $\alpha \in$ $(n-1, n)$, and $f^{(n)}(\tau)$ is the $n^{t h}$ order derivative of the function $f(\tau)$. For simplicity in the text we often write the Caputo fractional derivative as $\partial_{t}^{\alpha}[\cdot]$.

The Riesz fractional derivative is expressed as

$$
\frac{\partial^{\alpha}}{\partial|x|^{\alpha}} f(x)=-\frac{1}{2 \cos (\pi \alpha / 2)}\left[I_{+}^{-\alpha} f(x)+I_{-}^{-\alpha} f(x)\right], \quad 0<\alpha \leqslant 2,
$$

where

$$
\left\{\begin{array}{l}
I_{+}^{\alpha} f(x)=\frac{1}{\Gamma(\alpha)} \int_{-\infty}^{x}(x-\xi)^{\alpha-1} f(\xi) \mathrm{d} \xi \\
I_{-}^{\alpha} f(x)=\frac{1}{\Gamma(\alpha)} \int_{x}^{+\infty}(\xi-x)^{\alpha-1} f(\xi) \mathrm{d} \xi
\end{array}\right.
$$

\section{Acknowledgements}

Lihong Guo thanks for the financial support from China Scholarships Council (CSC), under No. 201806170154.

\section{References}

[1] E. Abbena, S. Salamon, A. Gray, Modern Differential Geometry of Curves and Surfaces with Mathematica. Chapman and Hall/CRC (2017); DOI: 10.1201/9781315276038. 
[2] A. Amir, An elementary renormalization-group approach to the generalized central limit theorem and extreme value distributions. J. Stat. Mech.: Theory Exp. 2020, No 1 (2020), 013214; DOI: 10.1088/1742-5468/ab5b8c

[3] D. J. Amit, V. Martin-Mayor, Field Theory, the Renormalization Group, and Critical Phenomena: Graphs to Computers. World Scientific Publishing Company (2005); DOI: $10.1142 / 5715$.

[4] P. W. Anderson, More is different. Science 177, No 4047 (1972), 393396.

[5] M. Andrews, M. Dinitz, Maximizing capacity in arbitrary wireless networks in the SINR model: Complexity and game theory. In: IEEE INFOCOM 2009, IEEE (2009), 1332-1340; DOI: 10.1109/INFCOM.2009.5062048.

[6] G. Arcioni, Using self-similarity and renormalization group to analyze time series. arXiv Preprint arXiv:0805.3213 (2008).

[7] V. I. Arnol'd, Small denominators and problems of stability of motion in classical and celestial mechanics. Russ. Math. Surv. 18, No 6 (1963), 85; DOI: 10.1070/RM1963v018n06ABEH001143.

[8] Y. Bar-Yam, Dynamics of Complex Systems. CRC Press (2019).

[9] A. L. Barabási, E. Bonabeau, Scale-free networks. Sci. Am. 288, No 5 (2003), 60-69; DOI: 10.1038/scientificamerican0503-60.

[10] E. Belbruno, Capture Dynamics and Chaotic Motions in Celestial Mechanics: With Applications to the Construction of Low Energy Transfers. Princeton University Press (2018).

[11] J. K. Bhattacharjee, S. Chakraborty, A. Sarkar, A methodology for classifying periodic orbits. In: Chaos Theory: Modeling, Simulation and Applications, World Scientific (2011), 120-126; DOI: 10.1142/9789814350341_0013.

[12] G. Bianconi, S. N. Dorogovstev, The spectral dimension of simplicial complexes: A renormalization group theory. J. Stat. Mech.: Theory Exp. 2020, No 1 (2020), 014005; DOI: 10.1088/1742-5468/ab5d0e.

[13] D. Blömker, C. Gugg, S. Maier-Paape, Stochastic Navier-Stokes equation and renormalization group theory. Phys. D 173, No 3-4 (2002), 137-152; DOI: 10.1016/S0167-2789(02)00621-8.

[14] N. N. Bogolyubov, D. V. Shirkov, Introduction to the Theory of Quantized Fields. Interscience Publishers (1960); DOI: 10.1063/1.3057034.

[15] J. W. Bruce, P. Giblin, Curves and Singularities: A Geometrical Introduction to Singularity Theory. Cambridge University Press, Cambridge (1992). 
[16] R. Bulla, T. A. Costi, T. Pruschke, Numerical renormalization group method for quantum impurity systems. Rev. Mod. Phys. 80, No 2 (2008), 395; DOI: 10.1103/RevModPhys.80.395.

[17] C. G. Callan Jr, Broken scale invariance in scalar field theory. Phys. Rev. D 2, No 8 (1970), 1541-1547; DOI: 10.1103/PhysRevD.2.1541.

[18] I. Calvo, J. C. Cuchí, J. G. Esteve, F. Falceto, Generalized central limit theorem and renormalization group. J. Stat. Phys. 141, No 3 (2010), 409-421; DOI: 10.1007/s10955-010-0065-y.

[19] I. Calvo, J. C. Cuchí, J. G. Esteve, F. Falceto, Extreme-value distributions and renormalization group. Phys. Rev. E 86, No 4 (2012), 041109; DOI: $10.1103 /$ PhysRevE.86.041109.

[20] L. Y. Chen, N. Goldenfeld, Renormalization-group theory for the propagation of a turbulent burst. Phys. Rev. A 45, No 8 (1992), 5572-5577; DOI: $10.1103 /$ PhysRevA.45.5572.

[21] L. Y. Chen, N. Goldenfeld, Numerical renormalization-group calculations for similarity solutions and traveling waves. Phys. Rev. E 51, No 6 (1995), 5577-5581; DOI: 10.1103/PhysRevE.51.5577.

[22] L. Y. Chen, N. Goldenfeld, Y. Oono, Renormalization-group theory for the modified porous-medium equation. Phys. Rev. A 44, No 10 (1991), 6544-6550; DOI: 10.1103/PhysRevA.44.6544.

[23] L. Y. Chen, N. Goldenfeld, Y. Oono, Renormalization group theory for global asymptotic analysis. Phys. Rev. Lett. 73, No 10 (1994), 13111315; DOI: 10.1103/PhysRevLett.73.1311.

[24] L. Y. Chen, N. Goldenfeld, Y. Oono, Renormalization group and singular perturbations: Multiple scales, boundary layers, and reductive perturbation theory. Phys. Rev. E 54, No 1 (1996), 376-394; DOI: 10.1103/PhysRevE.54.376.

[25] W. Chen, Y. Liang, X. Hei, Structural derivative based on inverse Mittag-Leffler function for modeling ultraslow diffusion. Fract. Calc. Appl. Anal. 19, No 5 (2016), 1250-1261; DOI: 10.1515/fca-2016-0064; https://www.degruyter.com/view/journals/fca/19/5/

fca.19.issue-5.xml.

[26] H. Chiba, $C^{1}$ approximation of vector fields based on the renormalization group method. SIAM J. Appl. Dyn. Syst. 7, No 3 (2008), 895-932; DOI: $10.1137 / 070694892$.

[27] H. Chiba, Extension and unification of singular perturbation methods for ODEs based on the renormalization group method. SIAM J. Appl. Dyn. Syst. 8, No 3 (2009), 1066-1115; DOI: 10.1137/090745957.

[28] Y. Chung, C. Jones, T. Schäfer, C. Wayne, Ultra-short pulses in linear and nonlinear media. Nonlinearity 18, No 3 (2005), 1351-1374; DOI: $10.1088 / 0951-7715 / 18 / 3 / 021$. 
[29] J. F. Crow, M. Kimura, An Introduction to Population Genetics Theory. The Blackburn Press, New Jersey (1970).

[30] J. H. Cushman, D. O'Malley, M. Park, Anomalous dispersion, renormalization groups, scaling laws and classification: A reflection on recent efforts. Adv. Water Resour. 62 (2013), 207-214; DOI: 10.1016/j.advwatres.2013.07.001.

[31] T. Deacon, S. Koutroufinis, Complexity and dynamical depth. Information 5, No 3 (2014), 404-423; DOI: 10.3390/info5030404.

[32] R. L. DeVille, A. Harkin, M. Holzer, K. Josić, T. J. Kaper, Analysis of a renormalization group method and normal form theory for perturbed ordinary differential equations. Phys. D 237, No 8 (2008), 1029-1052; DOI: $10.1016 /$ j.physd.2007.12.009.

[33] R. L. DeVille, A. Harkin, K. Josic, T. J. Kaper, Asymptotic normal form theory for nonautonomous equations and its connections with a renormalization group method. SIAM J. Appl. Dyn. Syst (2004), 1-43.

[34] D. C. Donderi, An information theory analysis of visual complexity and dissimilarity. Perception 35, No 6 (2006), 823-835; DOI: $10.1068 / \mathrm{p} 5249$

[35] J. F. Douglas, Surface-interacting polymers: An integral-equation and fractional-calculus approach. Macromolecules 22, No 4 (1989), 17861797; DOI: $10.1021 / \mathrm{ma00194a049}$.

[36] S. I. Ei, K. Fujii, T. Kunihiro, Renormalization-group method for reduction of evolution equations; invariant manifolds and envelopes. Ann. Phys. 280, No 2 (2000), 236-298; DOI: 10.1006/aphy.1999.5989.

[37] M. Eigen, J. McCaskill, P. Schuster, The molecular quasi-species. Adv. Chem. Phys. 75 (1989), 149-263; DOI: 10.1002/9780470141243.ch4.

[38] H. Fallahgoul, S. Focardi, F. Fabozzi, Fractional Calculus and Fractional Processes with Applications to Financial Economics: Theory and Application. Academic Press (2016).

[39] J. Feldman, E. Trubowitz, Perturbation theory for many fermion systems. Helv. Phys. Acta. 63, No 1/2 (1990), 157-260.

[40] M. E. Fisher, Renormalization group theory: Its basis and formulation in statistical physics. Rev. Mod. Phys. 70, No 2 (1998), 653-681; DOI: 10.1103/RevModPhys.70.653.

[41] E. Fradkin, Field Theories of Condensed Matter Physics. Cambridge University Press, Cambridge (2013); DOI: 10.1017/CBO9781139015509.

[42] P. Galanter, Complexism and the role of evolutionary art. In: The Art of Artificial Evolution, Springer (2008), 311-332; DOI: 10.1007/978-3-540-72877-1_15. 
[43] L. K. Gallos, C. Song, H. A. Makse, A review of fractality and selfsimilarity in complex networks. Phys. A 386, No 2 (2007), 686-691; DOI: $10.1016 /$ j.physa.2007.07.069.

[44] Y. Gefen, A. Aharony, Y. Shapir, B. B. Mandelbrot, Phase transitions on fractals. II. Sierpinski gaskets. J. Phys. A: Math. Gen. 17, No 2 (1984), 435; DOI: 10.1088/0305-4470/17/2/028.

[45] M. Gell-Mann, F. E. Low, Quantum electrodynamics at small distances. Phys. Rev. 95, No 5 (1954), 1300-1312; DOI: 10.1103/PhysRev.95.1300.

[46] J. E. Gillis, G. H. Weiss, Expected number of distinct sites visited by a random walk with an infinite variance. J. Math. Phys. 11, No 4 (1970), 1307-1312; DOI: $10.1063 / 1.1665260$.

[47] N. Glatt-Holtz, M. Ziane, Singular perturbation systems with stochastic forcing and the renormalization group method. Discrete 86 Continuous Dynamical Systems-A 26, No 4 (2010), 1241-1268; DOI: 10.3934/dcds.2010.26.1241.

[48] N. Goldenfeld, Lectures on Phase Transitions and the Renormalization Group. CRC Press (1992); DOI: 10.1201/9780429493492.

[49] N. Goldenfeld, O. Martin, Y. Oono, Asymptotics of partial differential equations and the renormalisation group. In: Asymptotics Beyond All Orders, Springer (1991), 375-383; DOI: 10.1007/978-1-4757-0435-8_30.

[50] R. Gorenflo, F. Mainardi, Fractional calculus and stable probability distributions. Arch. Mech. 50, No 3 (1998), 377-388.

[51] S. I. Goto, Y. Masutomi, K. Nozaki, Lie-group approach to perturbative renormalization group method. Prog. Theor. Phys. 102, No 3 (1999), 471-497; DOI: 10.1143/PTP.102.471.

[52] S. J. Guastello, M. Koopmans, D. Pincus, Chaos and Complexity in Psychology: The Theory of Nonlinear Dynamical Systems. Cambridge University Press, Cambridge (2008).

[53] E. A. Guggenheim, The principle of corresponding states. J. Chem. Phys. 13, No 7 (1945), 253-261; DOI: 10.1063/1.1724033.

[54] L. Guo, S. Shi, Y. Chen, Renormalization group method for singular perturbed systems driven by fractional Brownian motion. In: International Design Engineering Technical Conferences and Computers and Information in Engineering Conference, American Society of Mechanical Engineers 59292 (2019), V009T12A022; DOI: 10.1115/DETC2019-98258.

[55] H. Haken, Information and Self-organization: A Macroscopic Approach to Complex Systems. Springer Science \& Business Media (2006). 
[56] B. Hernández-Bermejo, Renormalization group approach to power-law modeling of complex metabolic networks. J. Theor. Biol. 265, No 3 (2010), 422-432; DOI: 10.1016/j.jtbi.2010.04.024.

[57] M. Holzer, T. J. Kaper, An analysis of the renormalization group method for asymptotic expansions with logarithmic switchback terms. Adv. Differ. Equat. 19, No 3/4 (2014), 245-282.

[58] K. Huang, A critical history of renormalization. Int. J. Mod. Phys. A 28, No 29 (2013), 1330050; DOI: 10.1142/S0217751X13300500.

[59] B. Hughes, E. Montroll, M. Shlesinger, Fractal random walks. J. Stat. Phys. 28, No 1 (1982), 111-126; DOI: 10.1007/BF01011626.

[60] G. Jona-Lasinio, Renormalization group and probability theory. Phys. Rep. 352, No 4-6 (2001), 439-458; DOI: 10.1016/S0370-1573(01)00042-4.

[61] L. P. Kadanoff, Scaling laws for Ising models near $T_{c}$. Physics 2, No 6 (1966), 263-272; DOI: 10.1103/PhysicsPhysiqueFizika.2.263.

[62] L. P. Kadanoff, W. Götze, D. Hamblen, et al., Static phenomena near critical points: Theory and experiment. Rev. Mod. Phys. 39, No 2 (1967), 395-431; DOI: 10.1103/RevModPhys.39.395.

[63] S. Kehrein, The Flow Equation Approach to Many-particle Systems, Springer (2007); DOI: $10.1007 / 3-540-34068-8$.

[64] A. A. Kilbas, H. M. Srivastava, J. J. Trujillo, Theory and Applications of Fractional Differential Equations, Elsevier (2006).

[65] E. Kirkinis, The renormalization group: A perturbation method for the graduate curriculum. SIAM Rev. 54, No 2 (2012), 374-388.

[66] A. N. Kolmogorov, A new metric invariant of transitive dynamical systems and automorphisms of Lebesgue spaces. Trudy Mat. Inst. Steklov 169, (1985), 94-98.

[67] T. Kunihiro, A geometrical formulation of the renormalization group method for global analysis. Prog. Theor. Phys. 94, No 4 (1995), 503514; DOI: 10.1143/PTP.94.503.

[68] T. Kunihiro, The renormalization-group method applied to asymptotic analysis of vector fields. Prog. Theor. Phys. 97, No 2 (1997), 179-200; DOI: 10.1143/PTP.97.179.

[69] W. Li, S. Shi, Singular perturbed renormalization group theory and its application to highly oscillatory problems. Discrete Contin. Dyn. Syst. 23, No 4 (2018), 1819-1833; DOI: 10.3934/dcdsb.2018089.

[70] C. Liu, The renormalization method based on the Taylor expansion and applications for asymptotic analysis. Nonlinear Dyn. 88, No 2 (2017), 1099-1124; DOI: 10.1007/s11071-016-3298-8.

[71] B. B. Mandelbrot, Fractals: Form, Chance and Dimension. W.H. Freeman and Company, San Francisco, CA (1977). 
[72] B. B. Mandelbrot, J. W. Van Ness, Fractional Brownian motions, fractional noises and applications. SIAM Rev. 10, No 4 (1968), 422-437; DOI: $10.1137 / 1010093$.

[73] A. Marciniak-Czochra, A. Mikelić, T. Stiehl, Renormalization group second-order approximation for singularly perturbed nonlinear ordinary differential equations. Math. Methods Appl. Sci. 41, No 14 (2018), 5691-5710; DOI: 10.1002/mma.5107.

[74] U. Merry, Coping with Uncertainty: Insights from the New Sciences of Chaos, Self-organization, and Complexity. Praeger Westport, CT (1995).

[75] I. Moise, R. Temam, Renormalization group method: Application to Navier-Stokes equation. Discrete Contin. Dyn. Syst. 6, No 1 (2000), 191-210; DOI: 10.3934/dcds.2000.6.191.

[76] I. Moise, M. Ziane, Renormalization group method. Applications to partial differential equations. J. Dyn. Differ. Equations 13, No 2 (2001), 275-321; DOI: 10.1023/A:1016680007953.

[77] F. J. Molz, H. Rajaram, S. Lu, Stochastic fractal-based models of heterogeneity in subsurface hydrology: Origins, applications, limitations, and future research questions. Rev. Geophys. 42, No 1 (2004), 1-42; DOI: 10.1029/2003RG000126.

[78] J. Moser, Stable and Random Motions in Dynamical Systems: With Special Emphasis on Celestial Mechanics. Princeton University Press, New Jersey (1973).

[79] B. Mudavanhu, R. E. O’Malley Jr, A new renormalization method for the asymptotic solution of weakly nonlinear vector systems. SIAM J. Appl. Math. 63, No 2 (2003), 373-397; DOI: 10.1137/S0036139901394311.

[80] K. Nakashima, Multiple existence of indefinite nonlinear diffusion problem in population genetics. J. Differ. Equations 268, No 12 (2020), 7803-7842; DOI: 10.1016/j.jde.2019.11.082.

[81] A. H. Nayfeh, Perturbation Methods. John Wiley \& Sons (1973).

[82] K. P. Nelson, S. Umarov, Nonlinear statistical coupling. Phys. A Stat. Mech. its Appl. 389, No 11 (2010), 2157-2163; DOI: 10.1016/j.physa.2010.01.044.

[83] K. P. Nelson, S. Umarov, M. A. Kon, On the average uncertainty for systems with nonlinear coupling. Phys. A Stat. Mech. its Appl. 468, (2017), 30-43; DOI: 10.1016/j.physa.2016.09.046.

[84] M. E. Newman, Power laws, Pareto distributions and Zipf's law. Contemp. Phys. 46, No 5 (2005), 323-351; DOI: 10.1080/00107510500052444. 
[85] M. E. Newman, Resource letter CS-1: Complex systems. Am. J. Phys. 79, No 8 (2011), 800-810; DOI: 10.1119/1.3590372.

[86] H. A. Orr, Adaptation and the cost of complexity. Evolution 54, No 1 (2000), 13-20; DOI: 10.1111/j.0014-3820.2000.tb00002.x.

[87] G. Pagnini, Y. Chen, Mellin convolution for signal filtering and its application to the Gaussianization of Lévy noise. In: Proc. of the ASME, Washington, DC (2011), 26-31; DOI: 10.1115/DETC2011-47392.

[88] A. Palit, D. P. Datta, Comparative study of homotopy analysis and renormalization group methods on Rayleigh and Van der Pol equations. Differ. Equ. Dyn. Syst. 24, No 4 (2016), 417-443; DOI: 10.1007/s12591-015-0253-y.

[89] L. Parker, D. J. Toms, Renormalization-group analysis of grand unified theories in curved spacetime. Phys. Rev. D 29, No 8 (1984), 1584-1608; DOI: 10.1103/PhysRevD.29.1584.

[90] L. Parrott, Measuring ecological complexity. Ecol. Indic. 10, No 6 (2010), 1069-1076; DOI: 10.1016/j.ecolind.2010.03.014.

[91] R. Peltier, J. L. Véhel, Multifractional Brownian motion: Definition and preliminary results (1995), RR-2645.

[92] M. E. Peskin, An Introduction to Quantum Field Theory. CRC Press (1995); DOI: 10.1201/9780429503559.

[93] M. Petcu, R. Temam, D. Wirosoetisno, Renormalization group method applied to the primitive equations. J. Differ. Equations 208, No 1 (2005), 215-257; DOI: $10.1016 /$ j.jde.2003.10.011.

[94] O. Pocovnicu, First and second order approximations for a nonlinear wave equation. J. Dyn. Differ. Equations 25, No 2 (2013), 305-333; DOI: $10.1007 / \mathrm{s} 10884-013-9286-5$.

[95] I. Podlubny, Fractional Differential Equations: An Introduction to Fractional Derivatives, Fractional Differential Equations, to Methods of Their Solution and Some of Their Applications. Academic Press, San Diego etc. (1999).

[96] H. Qian, Fractional Brownian motion and fractional Gaussian noise. In: Processes with Long-Range Correlations, Springer (2003), 22-33; DOI: $10.1007 / 3-540-44832-2 \_2$.

[97] W. J. Reed, The Pareto law of incomes - an explanation and an extension. Phys. A 319 (2003), 469-486;

DOI: 10.1016/S0378-4371(02)01507-8.

[98] S. Sachdev, Quantum phase transitions. Handb. Magn. Adv. Magn. Mater. (2007); DOI: 10.1002/9780470022184.hmm108.

[99] W. K. A. Salem, On the renormalization group approach to perturbation theory for PDEs. Ann. Henri Poincaré 11, No 6 (2010), 1007-1021; DOI: $10.1007 / \mathrm{s} 00023-010-0046-3$. 
[100] S. I. Sasa, Renormalization group derivation of phase equations. Phys. $D$ 108, No 1-2 (1997), 45-59; DOI: 10.1016/S0167-2789(97)82004-0.

[101] H. Schoeller, A perturbative nonequilibrium renormalization group method for dissipative quantum mechanics. Eur. Phys. J. Spec. Top. 168, No 1 (2009), 179-266; DOI: 10.1140/epjst/e2009-00962-3.

[102] H. Schoeller, J. König, Real-time renormalization group and charge fluctuations in quantum dots. Phys. Rev. Lett. 84, No 16 (2000), 3686; DOI: 10.1103/PhysRevLett.84.3686.

[103] H. G. Schuster, Reviews of Nonlinear Dynamics and Complexity. Wiley Online Library (2008).

[104] J. V. Sengers, J. G. Shanks, Experimental critical-exponent values for fluids. J. Stat. Phys. 137, No 5-6 (2009), 857-877; DOI: 10.1007/s10955-009-9840-z.

[105] H. Sheng, Y. Chen, T. Qiu, Tracking performance and robustness analysis of Hurst estimators for multifractional processes. IET signal processing 6, No 3 (2012), 213-226; DOI: 10.1049/iet-spr.2010.0170.

[106] D. V. Shirkov, Evolution of the Bogoluibov's renormalization group. arXiv preprint hep-th/9909024 (1999).

[107] J. Sólyom, The Fermi gas model of one-dimensional conductors. Adv. Phys. 28, No 2 (1979), 201-303; DOI: 10.1080/00018737900101375.

[108] C. Song, S. Havlin, H. A. Makse, Self-similarity of complex networks. Nature 433, No 7024 (2005), 392-395; DOI: 10.1038/nature03248.

[109] D. Sornette, Critical Phenomena in Natural Sciences: Chaos, Fractals, Selforganization and Disorder: Concepts and Tools. Springer Science \& Business Media (2006).

[110] E. Stueckelberg, A. Petermann, La renormalisation des constants dans la théorie de quanta. Helv. Phys. Acta. 26 (1953), 499-520; DOI: $10.5169 /$ seals-112426.

[111] H. Sun, Y. Zhang, D. Baleanu, W. Chen, Y. Chen, A new collection of real world applications of fractional calculus in science and engineering. Commun. Nonlinear Sci. Numer. Simul. 64 (2018), 213-231; DOI: 10.1016/j.cnsns.2018.04.019.

[112] H. Sun, R. M. Ziff, G. Bianconi, Renormalization group theory of percolation on pseudo-fractal simplicial and cell complexes. Phys. Rev. E 102 (2020), 012308; DOI: 10.1103/PhysRevE.102.012308.

[113] M. Suzuki, Phase transition and fractals. Prog. Theor. Phys. 69, No 1 (1983), 65-76; DOI: 10.1143/PTP.69.65.

[114] K. Symanzik, Small distance behaviour in field theory and power counting. Commun. Math. Phys. 18, No 3 (1970), 227-246; DOI: 10.1007/BF01649434. 
[115] Z. Tan, J. Zhang, J. Wang, J. Xu, Day-ahead electricity price forecasting using wavelet transform combined with ARIMA and GARCH models. Appl. Energy 87, No 11 (2010), 3606-3610; DOI: 10.1016/j.apenergy.2010.05.012.

[116] Y. Tao, Spontaneous economic order. J. Evol. Econ. 26, No 3 (2016), 467-500; DOI: 10.1007/s00191-015-0432-6.

[117] R. M. Temam, D. Wirosoetisno, On the solutions of the renormalized equations at all orders. Adv. Differ. Equat. 8, No 8 (2003), 1005-1024.

[118] S. Thurner, Nonextensive statistical mechanics and complex scalefree networks. Europhys. News 36, No 6 (2005), 218-220, DOI: 10.1051/epn:2005612.

[119] M. Troyer, U. Wiese, Computational complexity and fundamental limitations to fermionic quantum Monte Carlo simulations. Phys. Rev. Lett. 94, No 17 (2005), 170201; DOI: 10.1103/PhysRevLett.94.170201.

[120] C. Tsallis, Introduction to Nonextensive Statistical Mechanics: Approaching a Complex World. Springer Science \& Business Media (2009).

[121] C. Tsallis, Statistical mechanics for complex systems: On the structure of $q$-triplets. In: Physical and Mathematical Aspects of Symmetries, Springer (2017), 51-59; DOI: 10.1007/978-3-319-69164-0_7.

[122] S. Umarov, Continuous time random walk models associated with distributed order diffusion equations. Fract. Calc. Appl. Anal. 18, No 3 (2015), 821-837; DOI: 10.1515/fca-2015-0049; https://www.degruyter.com/view/journals/fca/18/3/

fca.18.issue-3.xml.

[123] A. B. Vasil'eva, V. F. Butuzov, L. V. Kalachev, The Boundary Function Method for Singular Perturbed Problems, Cambridge University Press, Cambridge (1987); DOI: 10.1137/1.9781611970784.

[124] L. Wang, First order approximation for quadratic dispersive equations by the renormalization group approach. J. Math. Phys. 55, No 12 (2014), 123503; DOI: 10.1063/1.4903001.

[125] F. Wegner, Flow-equations for Hamiltonians. Ann. Phys. 506, No 2 (1994), 77-91; DOI: 10.1002/andp.19945060203.

[126] B. J. West, Physiology, Promiscuity, and Prophecy at the Millennium: A Tale of Tails. World Scientific (1999); DOI: 10.1142/4069.

[127] B. J. West, Comments on the renormalization group, scaling and measures of complexity. Chaos, Solitons Fractals 20, No 1 (2004), 3344; DOI: 10.1016/S0960-0779(03)00425-9.

[128] B. J. West, Where Medicine Went Wrong: Rediscovering the Path to Complexity, World Scientific (2006).

[129] B. J. West, Nature's Patterns and the Fractional Calculus. Walter de Gruyter GmbH, Berlin/Boston (2017); DOI: 10.1515/9783110535136. 
[130] B. J. West, P. Grigolini, Complex Webs: Anticipating the Improbable. Cambridge University Press, Cambridge (2010).

[131] B. J. West, K. Mahmoodi, P. Grigolini, Empirical Paradox, Complexity Thinking and Generating New Kinds of Knowledge. Cambridge Scholars Publishing (2019).

[132] B. J. West, D. West, Fractional dynamics of allometry. Fract. Calc. Appl. Anal. 15, No 1 (2012), 70-96; DOI: 10.2478/s13540-012-0006-3; https://www.degruyter.com/view/journals/fca/15/1/ fca.15.issue-1.xml.

[133] K. G. Wilson, Renormalization group and critical phenomena. I. Renormalization group and the Kadanoff scaling picture. Phys. Rev. B 4, No 9 (1971), 3174-3183; DOI: 10.1103/PhysRevB.4.3174.

[134] K. G. Wilson, Renormalization group and critical phenomena. II. Phase-space cell analysis of critical behavior. Phys. Rev. B 4, No 9 (1971), 3184-3205; DOI: 10.1103/PhysRevB.4.3184.

[135] K. G. Wilson, The renormalization group: Critical phenomena and the Kondo problem. Rev. Mod. Phys. 47, No 4 (1975), 773-840; DOI: 10.1103/RevModPhys.47.773.

[136] K. G. Wilson, The renormalization group and critical phenomena. Rev. Mod. Phys. 55, No 3 (1983), 583-600; DOI: 10.1103/RevModPhys.55.583.

[137] S. L. Woodruff, The use of an invariance condition in the solution of multiple-scale singular perturbation problems: Ordinary differential equations. Stud. Appl. Math. 90, No 3 (1993), 225-248; DOI: 10.1002/sapm1993903225.

[138] S. L. Woodruff, A uniformly-valid asymptotic solution to a matrix system of ordinary differential equations and a proof of its validity. Stud. Appl. Math. 94, No 4 (1995), 393-413; DOI: 10.1002/sapm1995944393.

[139] Y. Y. Yamaguchi, Y. Nambu, Renormalization group equations and integrability in Hamiltonian systems. Prog. Theor. Phys. 100, No 1 (1998), 199-204; DOI: 10.1143/PTP.100.199.

[140] Y. Y. Yamaguchi, Y. Nambu, Renormalization group method and canonical perturbation theory. arXiv Preprint chao-dyn/9902013 (1999).

[141] Q. Yang, D. Chen, T. Zhao, Y. Chen, Fractional calculus in image processing: A review. Fract. Calc. Appl. Anal. 19, No 5 (2016), 12221249; DOI: 10.1515/fca-2016-0063; https://www.degruyter.com/view/journals/fca/19/5/

fca.19.issue-5.xml.

[142] V. I. Yukalov, Self-similar approach to market analysis. Eur. Phys. J. $B$ 20, No 4 (2001), 609-617; DOI: 10.1007/PL00011115. 
[143] G. Zaslavsky, Renormalization group theory of anomalous transport in systems with Hamiltonian chaos. Chaos 4, No 1 (1994), 25-33; DOI: 10.1063/1.166054.

[144] G. M. Zaslavsky, G. M. Zaslavskij, Hamiltonian Chaos and Fractional Dynamics. Oxford University Press, on Demand (2005).

[145] R. Zhou, S. Shi, W. Li, Renormalization group approach to boundary layer problems. Commun. Nonlinear Sci. Numer. Simul. 71 (2019), 220-230; DOI: 10.1016/j.cnsns.2018.11.012.

[146] M. Ziane, On a certain renormalization group method. J. Math. Phys. 41, No 5 (2000), 3290-3299; DOI: 10.1063/1.533307.

[147] B. J. Zubillaga, A. L. Vilela, C. Wang, K. P. Nelson, H. E. Stanley, A three-state opinion formation model for financial markets. arXiv Preprint arXiv:1905.04370 (2019).

[148] B. J. Zubillaga, The Statistical Mechanics of Societies: Opinion Formation Dynamics and Financial Markets. Doctoral Dissertation, Boston University (2020).

${ }^{1}$ Institute of Mathematics

Jilin University, Changchun 130012, CHINA

e-mail: guolh17@mails.jlu.edu.cn

e-mail: shisy@jlu.edu.cn

2 Mechatronics, Embedded Systems and

Automation Laboratory, Dept. of Engineering

University of California, Merced, CA 95343, USA

e-mail: ychen53@ucmerced.edu (Corresponding author)

Received: July 31, 2020

3 Center for Studies of Nonlinear Science

University of North Texas, Denton, TX, USA

e-mail: brucejwest213@gmail.com

Please cite to this paper as published in:

Fract. Calc. Appl. Anal., Vol. 24, No 1 (2021), pp. 5-53,

DOI: $10.1515 /$ fca-2021-0002 